\title{
A Novel Fractional Tikhonov Regularization Coupled with an Improved Super-Memory Gradient Method and Application to Dynamic Force Identification Problems
}

\author{
Nengjian Wang, ${ }^{1}$ Chunping Ren $\mathbb{D}^{1},{ }^{1}$ and Chunsheng Liu ${ }^{2}$ \\ ${ }^{1}$ College of Mechanical and Electrical Engineering, Harbin Engineering University, Harbin 150001, China \\ ${ }^{2}$ School of Mechanical Engineering, Heilongjiang University of Science and Technology, Harbin 150022, China \\ Correspondence should be addressed to Chunping Ren; renchunpin@sina.com
}

Received 8 January 2018; Revised 27 March 2018; Accepted 15 April 2018; Published 2 July 2018

Academic Editor: Kishin Sadarangani

Copyright (c) 2018 Nengjian Wang et al. This is an open access article distributed under the Creative Commons Attribution License, which permits unrestricted use, distribution, and reproduction in any medium, provided the original work is properly cited.

\begin{abstract}
This paper presents a novel inverse technique to provide a stable optimal solution for the ill-posed dynamic force identification problems. Due to ill-posedness of the inverse problems, conventional numerical approach for solutions results in arbitrarily large errors in solution. However, in the field of engineering mathematics, there are famous mathematical algorithms to tackle the illposed problem, which are known as regularization techniques. In the current study, a novel fractional Tikhonov regularization (NFTR) method is proposed to perform an effective inverse identification, then the smoothing functional of the ill-posed problem processed by the proposed method is regarded as an optimization problem, and finally a stable optimal solution is obtained by using an improved super-memory gradient (ISMG) method. The result of the present method is compared with that of the standard TR method and FTR method; new findings can be obtained; that is, the present method can improve accuracy and stability of the inverse identification problem, robustness is stronger, and the rate of convergence is faster. The applicability and efficiency of the present method in this paper are demonstrated through a mathematical example and an engineering example.
\end{abstract}

\section{Introduction}

In this paper, we present a novel regularization technique to solve the following linear operator equations of the form:

$$
\mathrm{Cx}=\mathbf{y},
$$

where $\mathbf{x}, \quad \mathbf{y} \in \mathfrak{R}^{n}$, and $\mathbf{C} \in \mathfrak{R}^{n \times n}$ represent the identified vector, the measured vector, and a compact linear operator, respectively;

Under these circumstances, a generalized solution of (1) can be obtained

$$
\mathbf{x}=\mathbf{C}^{\dagger} \mathbf{y}
$$

where $\mathbf{C}^{\dagger}$ is the Moore-Penrose pseudoinverse of $\mathbf{C}$ which is usually an ill-conditioned operator, as, for example, it could be if $\operatorname{det} C=0$, which causes (2) to be a typical ill-posed problem; see $[1,2]$.
Ill-posed problem exists widely in many fields of science and has important application in engineering [3]. However, in many cases of linear equation which appears in practical engineering problem, generally, it is clear that the measured vector $\mathbf{y}$ is rarely obtained exactly. Conversely, noises are contained in $\mathbf{y}$ owing to the measurement error. Consequently, when $\mathbf{C}$ is severely ill-conditioned and $\mathbf{y}$ is disturbed through noise, we will be confronted with a problem in which the generalized solution of an ill-posed linear equation may be seriously deviated from the exact solution [4-6].

In mathematical theory, there are several typical regularization methods which can solve the ill-posed problem in practical engineering applications. For example, the standard Tikhonov regularization (TR) method, as a traditional technique, has been widely adopted for overcoming the ill-posed nature (or numerical instability), such as system parameter identification, load source identification problem, and so on [7-10]. T. S. Jang et al. [11] applied the standard TR method to identify the loading source of infinite beams 
on an elastic foundation from given information of vertical deflection of infinite beams. J. Gao et al. [12] utilized the standard TR method for identifying the unknown load source. V. Melicher et al. [13] identified the piecewise constant parameter using the standard TR method. In addition, many works are mainly concerned with the identification of load position and load number in nonlinear system using the standard TR method [14-16]. However, the standard TR method is not completely perfect; there are some unavoidable limitations and disadvantages: (i) the standard TR method may determine approximate solutions that are too smooth; (ii) the approximate solution may lack many details that the desired exact solution might possess; therefore, it is very necessary to discuss other regularization methods to improve the limitations and disadvantages of the regularization method mentioned above.

In the past few years, fractional Tikhonov regularization (FTR) method was gradually introduced to deal with the ill-posed problems mentioned above [17]. Furthermore, the FTR method has been applied to many fields and obtained some remarkable achievements [18-20]. M. E. Hochstenbach et al. [21] used the FTR method to obtain approximate solution of higher quality which is better than the standard TR method in solving linear system equation with a severe ill-posedness. In [22], we can see that the FTR method is significantly better than the standard TR method, which was proved by several numerical examples. It can be found from [23] that the FTR method can obtain more approximation solutions than the standard TR method, which was discussed through several computed examples. J. Zhang et al. [24] illustrated that the FTR method was more suitable for tackling partly textured images problems. Hence, the FTR method mentioned above has many advantages, to a certain extent, which may not only solve the shortcomings of the standard TR method, but also be able to provide a useful help for obtaining the effective solution of the ill-posed problem. However, FTR method is also not very perfect, which has great limitations for solving the ill-posed problem. First, the solutions of the larger singular values can not be retained well. Then it is not completely effective for the large-scale ill-posed problem. Thus, it is very necessary to discuss an effective technique to tackle the limitations of the problem encountered.

Considering the limitations of TR method and FTR method in a certain case, we propose a novel fractional Tikhonov regularization (NFTR) method to offer a stable solution for the ill-posed problems in practical engineering applications. In our work, the ill-posed problem processed by the proposed method is redefined as a class of unconstrained optimization problems to solve the following minimum problem:

$$
\min _{\mathbf{x} \in \Re^{n}} f(\mathbf{x})=\left.\Lambda\right|_{\mathrm{NFTR}} .
$$

In which $f(\mathbf{x}): \mathfrak{R}^{n} \longrightarrow \mathfrak{R}$ is a continuously differentiable function, and $\left.\Lambda\right|_{\text {NFTR }}$ denotes the smoothing functional using the NFTR method.

In fact, there are many optimization algorithms that can improve the efficiency of solving (3), like conjugate gradient (CG) method, memory gradient (MG) method, super-memory gradient (SMG) method, and so on [25-27]. It is generally known that CG method does not achieve global convergence for common functions under inexact line searches [28], whereas MG method requires dealing with the trust region subproblem [29]. Although SMG method is superior to $\mathrm{CG}$ and $\mathrm{MG}$ method, in some cases, it is also not globally convergent [30]. Therefore, it is very meaningful to find a global convergence algorithm based on the SMG method. Hence, in this paper, an improved supermemory gradient (ISMG) method is introduced to deal with unconstrained optimization problems of (3).

This article is focused on a novel stable inverse technique developed to provide an optimal solution for the ill-posed problems in practical engineering. Compared with our previous works, some highlights and advances of the proposed technique are derived in this article.

First of all, the article is different from our previous studies, and the highlights of this article are described as follows: (i) a novel fractional Tikhonov regularization (NFTR) coupled with an improved super-memory gradient (ISMG) method is proposed; (ii) solving the ill-posed problem procedure is regarded as a class of unconstrained optimization problems; (iii) the detailed information of the solution is clearly enhanced; (iv) the technique we propose can effectively overcome the smoothness of the solution of the ill-posed problem; (v) global convergence and stability of the solution are proved.

Then, some advances of the proposed technique are described as follows: (i) a stable optimal solution for the illposed dynamic force identification problems can be obtained; (ii) the proposed method yields approximate solutions of higher quality than the standard TR method and FTR method; (iii) the technique proposed does not need any a priori information on the model of the inverse identification; only the measured response is sufficient for the present method; (iv) the applicability of the present technique is examined through a mathematical example and an engineering example.

In this paper we propose a novel fractional Tikhonov regularization (NFTR) coupled with an improved supermemory gradient (ISMG) method to solve the ill-posed dynamic force identification problems in engineering applications. The remainder of this paper is formulated as follows. The inverse problem model is described in Section 2. In Section 3, we present the NFTR method for solving the illposed problem. In Section 4, ISMG method is introduced to deal with an optimization problem. The convergence properties of the present method are proved in Section 5. In Section 6, we discuss the application of a mathematical and an engineering example. Section 7 shows some meaningful findings and new conclusions.

\section{Description of Inverse Problem Model}

The model of inverse problems encountered in science and engineering can be uniformly expressed by a Fredholm integral equation of the first kind [31-33]: 


$$
\int_{\mathrm{a}}^{\mathrm{b}} \mathrm{c}(t-\tau) \mathrm{x}(\tau) \mathrm{d} \tau=\mathrm{y}(t)
$$

where $\mathrm{x}(\mathrm{t}), \mathrm{c}(\mathrm{t}-\tau)$, and $\mathrm{y}(\mathrm{t})$ represent the identified object, the kernel function, and the measured response, respectively.

The discretization form of (4) is processed based on the rectangular formula

$$
\sum_{i=1}^{n} \mathrm{c}\left(t_{k}-\tau_{i}\right) \mathrm{x}\left(\tau_{i}\right) \Delta \mathrm{T}=\mathrm{y}\left(t_{k}\right)
$$

in which $\Delta \mathrm{T}$ is time interval; we consider that

$$
\begin{aligned}
\mathrm{x}_{i} & =\mathrm{x}\left(\tau_{i}\right), \\
\mathrm{y}_{k} & =\mathrm{y}\left(t_{k}\right), \\
\mathrm{c}_{k-i} & =\mathrm{c}\left(t_{k}-\tau_{i}\right) .
\end{aligned}
$$

Then we have

$$
\begin{aligned}
& \mathbf{x}=\left(\mathrm{x}_{1}, \mathrm{x}_{2}, \mathrm{x}_{3}, \cdots \mathrm{x}_{n}\right)^{\mathrm{T}}, \\
& \mathbf{y}=\left(y_{1}, \mathrm{y}_{2}, \mathrm{y}_{3}, \cdots \mathrm{y}_{n}\right)^{\mathrm{T}}, \\
& \mathbf{C}=\left(c_{k-i}\right) \Delta \mathrm{T} .
\end{aligned}
$$

Hence, (5) can be simply written as

$$
\mathbf{C x}=\mathbf{y}
$$

The vector $y$ represents available data, which is contaminated by an error $e$. The error may stem from measurement inaccuracies or discretization. Thus,

$$
\mathbf{y}=\widehat{\mathbf{y}}+e
$$

where $\hat{y}$ is the unknown error-free vector associated with $y$. We will assume the unavailable error-free system

$$
\mathbf{C x}=\widehat{\mathbf{y}}
$$

to be consistent and denote its solution of minimal Euclidean norm by $\hat{x}$. We would like to determine an approximation of $\hat{x}$ by computing a suitable approximate solution of (10). Due to the ill-conditioning of the matrix $C$ and the error $e$ in $y$, the solution of the least-squares problem (10) of minimal Euclidean norm is typically a poor approximation of $\widehat{x}$, and a small perturbation in $y$ may give rise to an arbitrarily large perturbation in $\hat{x}$, or even make the problem unsolvable. Moreover, the right-hand side function that is available in applications represents data that is contaminated by noise. Thus, instead of $\hat{y}$, the error-contaminated function $y$ is available $[34,35]$. Therefore, it is very necessary to find an effective method to solve the ill-posed problem.

\section{The Regularization Method for Solving The Ill-Posed Problem}

In some instances, we all know that some traditional numerical methods are not able to solve (8) effectively. However, the regularization method, as a way to deal with the illposed problem, has been used by mathematical workers and engineering technicians [36-38]. In the past few years, the standard Tikhonov regularization (TR) and fractional Tikhonov regularization (FTR) method have been successfully applied to many engineering fields. Although many meaningful achievements have been made, there are still many limitations. Hence, in this work, considering the disadvantages of the standard TR method and FTR method, a novel fractional Tikhonov regularization (NFTR) method is discussed.

3.1. The Standard Tikhonov Regularization (TR) Method. The standard Tikhonov regularization (TR) method is a popular approach to determine an approximation of x; see, e.g., [3941] for properties and applications. This method replaces the minimization problem (8) by a penalized least-squares problem of the following form [42]:

$$
\min f(\mathbf{x})=\|\mathbf{y}-\mathbf{C} \mathbf{x}\|^{2}+\left\|\mathbf{A}_{\mu} \mathbf{x}\right\|^{2},
$$

in which $\mathbf{A}_{\mu}=\mu \mathbf{I}$, and $\mathbf{A}_{\mu}, \mu$, and $\mathbf{I}$ represent the regularization matrix, the regularization parameter, and the unit matrix, respectively.

3.2. Fractional Tikhonov Regularization (FTR) Method. It is well known that Tikhonov regularization in standard form typically determines a regularized solution that is too smooth; i.e., many details of the desired solution are not represented by the regularized solution. This shortcoming led Klann and Ramlau to introduce the fractional Tikhonov regularization method [17]. Subsequently another approach, also referred to as fractional Tikhonov regularization (FTR), was investigated in [21], the model of which is described as follows:

$$
\min f(\mathbf{x})=\|\mathbf{y}-\mathbf{C} \mathbf{x}\|_{\mathbf{W}}^{2}+\mu^{2}\|\mathbf{x}\|_{2}^{2} .
$$

Then we know

$$
\|\mathbf{x}\|_{\mathbf{W}}^{2}=\mathbf{x}^{\mathrm{T}} \mathbf{W} \mathbf{x}
$$

In which $\mathbf{W}$ is defined with the aid of the Moore-Penrose pseudoinverse of $C C^{T}$.

$$
\mathbf{W}=\left(\mathbf{C C}^{\mathrm{T}}\right)^{(\alpha-1) / 2}
$$

where $\alpha$ denotes fractional order and $\alpha \in(0,1) . C^{\mathrm{T}}$ denotes the transpose of $C$. It is obvious that FTR method is different than that of TR method; it mainly considers the influence of fractional order on the identified objects, which thus may be more suitable for solving the ill-posed identification problem. However, FTR method is not perfect, which has great limitations for solving the ill-posed problem. First, the solutions of the larger singular values can not be retained well. Then it is not completely effective for solving the largescale ill-posed problem. Thus, it is very necessary to find an effective method to solve the limitations of problem we encountered. 
3.3. A Novel Fractional Tikhonov Regularization (NFTR) Method. From Sections 3.1 and 3.2, on the one hand, we can find that the regular solution is obtained by using the standard TR method that may be too smooth and may lose some details of the solution we expected. On the other hand, the solutions of the larger singular values can not be retained perfectly using the FTR method for dealing with some practical complex engineering applications. Hence, in order to solve the limitations of TR method and FTR method in a certain case, in this paper, a novel fractional Tikhonov regularization (NFTR) method is proposed. The model of the proposed method is described as follows:

$$
\min f(\mathbf{x})=\|\mathbf{y}-\mathbf{C} \mathbf{x}\|_{\mathbf{M}}^{2}+\left\|\mathbf{S}_{\mu} \mathbf{x}\right\|_{2}^{2},
$$

in which

$$
\begin{aligned}
\|\mathbf{x}\|_{\mathbf{M}}^{2} & =\frac{1}{2} \mathbf{x}^{\mathrm{T}} \mathbf{M} \mathbf{x} \\
\mathbf{M} & =\left(\mathbf{C} C^{\mathrm{T}}\right)^{(\alpha-1) / 2} \\
\mathbf{S}_{\mu} & =\Gamma \mathbf{N}_{\mu} \mathbf{V}^{\mathrm{T}}
\end{aligned}
$$

where $\mathbf{S}_{\mu}, \mathbf{N}_{\mu}, \mathbf{V}^{\mathrm{T}}, \Gamma$, and $\mathbf{M}$ represent the regularization matrix, the diagonal matrix, right column orthogonal matrix of singular value decomposition of $\mathbf{C}$, adjustment coefficient matrix, and symmetric positive semidefinite matrix, respectively. In this work, $\Gamma$ is arranged as the identity matrix; that is, $\Gamma=I_{n}=\operatorname{diag}(1,1, \ldots, 1)$, in which $\mathbf{N}_{\mu}$ can be described as follows:

$$
\mathbf{N}_{\mu}=\left[\begin{array}{llll}
\max \left\{\mu^{2}-\sigma_{1}{ }^{2}, 0\right\} & & & \\
& \max \left\{\mu^{2}-\sigma_{2}{ }^{2}, 0\right\} & & \\
& \ddots & \\
& & \max \left\{\mu^{2}-{\sigma_{n}}^{2}, 0\right\}
\end{array}\right] .
$$

Hence, the NFTR method corresponding to the normal equation can be expressed in the following form:

$$
\left(\left(\mathrm{C}^{\mathrm{T}} \mathbf{C}\right)^{(\alpha+1) / 2}+\mathbf{S}_{\mu}^{\mathrm{T}} \mathbf{S}_{\mu}\right) \mathbf{x}=\left(\mathrm{C}^{\mathrm{T}} \mathbf{C}\right)^{(\alpha+1) / 2} \mathrm{C}^{\mathrm{T}} \mathbf{y}
$$

where $S_{\mu}^{\mathrm{T}}$ denotes the transpose of $S_{\mu}$.

Introduce the singular value decomposition (SVD) [43]:

$$
\mathbf{C}=\mathbf{U} \Sigma \mathbf{V}^{\mathrm{T}}=\sum_{i=1}^{n} u_{i} \sigma_{i} v_{i}^{T},
$$

in which $\mathbf{U}=\left(u_{1}, u_{2}, u_{3}, \cdots u_{n}\right) \in \mathfrak{R}^{n \times n}$ and

$$
\mathbf{V}=\left(v_{1}, v_{2}, v_{3}, \cdots v_{n}\right) \in \mathfrak{R}^{n \times n} \text { are orthogonal matrices, }
$$
and

$$
\Sigma=\operatorname{diag}\left(\sigma_{1}, \sigma_{2}, \sigma_{3}, \cdots \sigma_{n}\right) \in \mathfrak{R}^{n \times n} .
$$

The singular values are ordered according to

$$
\sigma_{1} \geq \sigma_{2} \geq \sigma_{3} \geq \cdots \geq \sigma_{r}>\sigma_{r+1}=\ldots=\sigma_{n}=0,
$$

in which the index $r$ is the rank of $C$; see, e.g., [43] for discussions on properties and the computation of the SVD.

Combining (18) and (19), then we have

$$
\left(\left(\Sigma^{\mathrm{T}} \Sigma\right)^{(\alpha+1) / 2}+\mathbf{S}_{\mu}^{2}\right) \mathbf{V}^{\mathrm{T}} \mathbf{x}=\left(\Sigma^{\mathrm{T}}\right)^{\alpha} \mathbf{U}^{\mathrm{T}} \mathbf{y} .
$$

Hence, we can obtain the regular solution $\mathbf{x}_{y s}$ of (22):

$$
\mathbf{x}_{y s}=\mathbf{V}\left(\left(\Sigma^{\mathrm{T}} \Sigma\right)^{(\alpha+1) / 2}+\mathbf{S}_{\mu}^{2}\right)^{-1}\left(\Sigma^{\mathrm{T}}\right)^{\alpha} \mathbf{U}^{\mathrm{T}} \mathbf{y}
$$

Meanwhile, the filter factor of TR, FTR, and NFTR method can be expressed as follows:

$$
\begin{aligned}
\Omega_{T R}\left(\sigma_{i}\right) & =\frac{\sigma_{i}^{2}}{\sigma_{i}^{2}+\mu^{2}}, \\
\Omega_{F T R}\left(\sigma_{i}\right) & =\frac{\sigma_{i}^{\alpha+1}}{\sigma_{i}^{\alpha+1}+\mu^{2}}, \\
\Omega_{N F T R}\left(\sigma_{i}\right) & = \begin{cases}1, & 1 \leq i \leq k, \\
\frac{\sigma_{i}^{\alpha+1}}{\mu^{2}} & k<i \leq n .\end{cases}
\end{aligned}
$$

3.4. Stability Analysis. In the paper, we may determine a suitable regular parameter $\mu>0$ by the discrepancy principle $[44,45]$; we choose $\mu>0$ so that

$$
\left\|\mathbf{C} \mathbf{x}_{\mathrm{ys}}-\mathbf{y}\right\|_{2}=\omega \ell
$$

where $\ell$ is equivalent to the noise level $\delta$, which satisfies $\|e\|_{2} \leq$ $\ell=\delta, e$ is the noise vector. $\omega$ is a user-supplied constant independent of $\ell$, and $\omega>1$.

Using (23) and (24), we have

$$
\begin{aligned}
\left\|\mathbf{x}_{\mathbf{y s}}\right\|_{2}^{2} & =\sum_{i=1}^{n} \frac{\Omega_{N F T R}^{2}\left(\sigma_{i}\right)\left(u_{i}^{T} \mathbf{y}\right)^{2}}{\sigma_{i}^{2}} \\
& =\sum_{i=1}^{k} \frac{\left(u_{i}^{T} \mathbf{y}\right)^{2}}{\sigma_{i}^{2}}+\sum_{i=k+1}^{n} \frac{\sigma_{i}^{2 \alpha+2}\left(u_{i}^{T} \mathbf{y}\right)^{2}}{\mu^{4} \sigma_{i}^{2}} .
\end{aligned}
$$


Finding the partial derivative of $\mu$ and $\alpha$ in (26), respectively, then

$$
\begin{aligned}
& \frac{\partial}{\partial \mu}\left\|\mathbf{x}_{\mathbf{y s}}\right\|_{2}^{2}=-4 \sum_{i=k+1}^{n} \frac{\sigma_{i}^{2 \alpha}\left(u_{i}^{T} \mathbf{y}\right)^{2}}{\mu^{5}}, \\
& \frac{\partial}{\partial \alpha}\left\|\mathbf{x}_{\mathbf{y s}}\right\|_{2}^{2}=2 \sum_{i=k+1}^{n} \frac{\sigma_{i}^{2 \alpha} \ln \sigma\left(u_{i}^{T} \mathbf{y}\right)^{2}}{\mu^{4}} .
\end{aligned}
$$

We can find from (27) that $\mu \longrightarrow\left\|\mathbf{x}_{\mathbf{y s}}\right\|_{2}^{2}$ is a monotonous decreasing function. When $\|\mathbf{C}\|<1$, then $\ln \sigma<0$, so we can obtain $\alpha \longrightarrow\left\|\mathbf{x}_{\mathbf{y s}}\right\|_{2}^{2}$, also a monotonous decreasing function.

However, it is clearly seen from (27) that the regularization parameter becomes large and the norm of regular solution becomes small. Due to $\alpha \longrightarrow\left\|\mathbf{x}_{\mathrm{ys}}\right\|_{2}^{2}$ being a monotonous decreasing function, so reducing $\alpha$ can increase the norm of regular solution according to $\alpha<1$.

Theorem 1. For $\ell>0$, the regularization parameter $\mu=\mu(\ell)$ meets (25), and then one has

$$
\frac{d \mu}{d \ell}>0
$$

Proof. The following form can be found:

$$
\left\|\mathbf{C} \mathbf{x}_{\mathbf{y s}}-\mathbf{y}^{d}\right\|_{2}^{2}=\sum_{i=1}^{n}\left(1-\Omega_{N F T R}\left(\sigma_{i}\right)\right)^{2}\left(u_{i}^{T} \mathbf{y}\right)^{2} .
$$

Then, the following form is obtained by substituting (24) and (25) into (29).

$$
\omega^{2} \ell(\mu)^{2}=\sum_{i=1}^{n}\left(1-\frac{\sigma_{i}^{\alpha+1}}{\mu^{2}}\right)^{2}\left(u_{i}^{T} \mathbf{y}\right)^{2} .
$$

Offering the derivative of $\mu$

$$
2 \omega^{2} \ell(\mu) \frac{d \ell}{d \mu}=2 \sum_{i=1}^{n}\left(1-\frac{\sigma_{i}^{\alpha+1}}{\mu^{2}}\right) \frac{\sigma_{i}^{\alpha+1}}{\mu^{3}}\left(u_{i}^{T} \mathbf{y}\right)^{2}
$$

We can see from the previous formula that $d \ell / d \mu>0$; then

$$
\frac{d \mu}{d \ell}>0
$$

Hence, the conclusion is proved.

In this paper, the NFTR method is of order optimal when the regularization parameter is chosen according to the discrepancy principle, and the proof process is shown as follows.

Let $A^{\mathrm{T}} A=\left(C^{\mathrm{T}} C\right)^{-\gamma}, S_{\mu}^{\mathrm{T}} S_{\mu}=\mu I$; then (18) can be described as a normal equation:

$$
\left(A^{\mathrm{T}} A+\mu\left(A^{\mathrm{T}} A\right)^{-\gamma}\right) \mathbf{x}_{\mathbf{y s}}=A^{\mathrm{T}} \mathrm{y}
$$

where $A^{\mathrm{T}}$ and $S_{\mu}^{\mathrm{T}}$ denote the transpose of $A$ and $S_{\mu}^{\mathrm{T}}, \gamma=(\alpha-$ $1) / 2$.
For our analysis, we assume a Hölder-type smoothness assumption, i.e., that the minimal norm solution $x_{y s}$ of the error-free problem (1) satisfies a smoothness condition of the form, for some constant $z$,

$$
\widehat{\mathrm{x}} \in R\left(\left(C^{\mathrm{T}} C\right)^{\vartheta}\right)
$$

With $\|\widehat{\mathrm{x}}\|_{\vartheta}:=\left(\sum_{n \geq 1} \sigma_{i}^{-2 \vartheta}\left|\left\langle\mathrm{x}, u_{n}\right\rangle\right|^{2}\right)^{1 / 2} \leq z$.

Here $\left(\sigma_{n} ; u_{n}, v_{n}\right)_{n \geq 1}$ is the singular system of the operator C. $\vartheta$ is a parameter which controls the a priori assumptions on the regularity of the domain set.

Theorem 2 ([17, Theorem 3.1]). Let $y \in \operatorname{range}(C)$ and $\| y-$ $y^{d} \| \leq \delta . \Omega_{N F T R}(\sigma)$ is a regularizing filter and assume that for $0 \leq \vartheta \leq \vartheta^{*}$,

$$
\sup _{0<\sigma \leq \sigma_{1}} \sigma^{-1}\left|\Omega_{N F T R}(\sigma)\right| \leq \kappa \mu^{-\varpi}
$$

and

$$
\sup _{0<\sigma \leq \sigma_{1}} \sigma^{\vartheta^{*}}\left|1-\Omega_{N F T R}(\sigma)\right| \leq \kappa_{\vartheta^{*}} \mu^{\varrho 9^{*}},
$$

where $\omega>0$ and $\kappa, \kappa_{9^{*}}$ are constants independent of $\delta$. The definition of $\vartheta^{*}$ is the same as that of $\vartheta$. Then with the a priori parameter choice

$$
\mu=p\left(\frac{\delta}{z}\right)^{(1 / \omega)(\vartheta+1)}, \quad p>0 \text { fixed. }
$$

The NFTR method induced by the filter $\Omega_{\text {NFTR }}(\sigma)$ is order optimal for all $0 \leq \vartheta \leq \vartheta^{*}$.

Lemma 3. The regularizing filter $\Omega_{N F T R}(\sigma)$ from (24) with $-1 \leq \alpha \leq 1$ fulfills Theorem 2 with $\omega=1 /(\alpha+1)$ and $\vartheta^{*}=\alpha+1$.

Proof. The filter $\Omega_{\text {NFTR }}(\sigma)$ is continuous on $(0, \infty)$. The regularizing properties of the filter $\Omega_{N F T R}(\sigma)$ are easily verified. One sees that

$$
\begin{aligned}
& \lim _{\sigma \longrightarrow 0} \sigma^{-1}\left|\Omega_{\text {NFTR }}(\sigma)\right|=\lim _{\sigma \longrightarrow \infty} \sigma^{-1}\left|\Omega_{N F T R}(\sigma)\right|=0, \\
& \lim _{\sigma \longrightarrow 0}\left|1-\Omega_{N F T R}(\sigma)\right| \sigma^{\vartheta}=0, \\
& \lim _{\sigma \longrightarrow \infty}\left|1-\Omega_{N F T R}\left(\sigma_{i}\right)\right| \sigma^{\vartheta}= \begin{cases}\infty & \vartheta>\alpha+1, \\
1 & \vartheta=\alpha+1, \\
0 & \vartheta<\alpha+1 .\end{cases}
\end{aligned}
$$

Hence, as long as $\vartheta<\alpha+1$, the suprema in Theorem 2 are attained as local maximal, which can be derived by simple calculus. One obtains

$$
\sup _{0<\sigma \leq \sigma_{1}} \sigma^{-1}\left|\Omega_{N F T R}(\sigma)\right| \leq \kappa \mu^{-1 /(\alpha+1)}
$$

and

$$
\sup _{0<\sigma \leq \sigma_{1}}\left|1-\Omega_{N F T R}(\sigma)\right| \sigma^{\vartheta^{*}} \leq \kappa_{\vartheta^{*}} \mu^{(1 /(\alpha+1)) \vartheta} .
$$

Consequently, by Theorem 2, the NFTR method (15) is order optimal for $-1<\alpha \leq 1$ and $0 \leq \vartheta \leq \alpha+1$. 


\section{The Algorithm for Solving Unconstrained Optimization Problem}

In general, it has always been known that the regular solution of (15) may give rise to an arbitrarily large perturbation in actual solution. However, one of the highlights of this work is expressed as follows: (15) is deemed considered as an unconstrained optimization problem, and the optimal solution of (15) can be obtained by using some optimization algorithms. In this paper, an improved super-memory gradient (ISMG) method was introduced to solve objective function of (15). Most of the well-known iterative algorithms for solving (15) take the following form [46-49]:

$$
x_{k+1}=x_{k}+h_{k} d_{k}
$$

where $d_{k}$ is the search direction.

In this paper, a new search direction $d_{k}$ of ISMG method is determined as follows:

$$
d_{k}= \begin{cases}-g_{k}, & k<m \\ -g_{k}+\sum_{i=1}^{m} \beta_{k}^{i} d_{k-i}, & k \geq m+1,\end{cases}
$$

where $g_{k}$ denotes the gradient of $f\left(x_{k}\right)$ at the point $x_{k}$, and $g_{k}=\nabla f\left(x_{k}\right) \cdot \beta_{k}^{i}$ is defined as follows:

$$
\beta_{k}^{i}=\frac{\rho\left\|g_{k}\right\|}{\left\|d_{k-i}\right\|}, \quad \rho \in\left(0, \frac{1}{m}\right) .
$$

$h_{k}$ of (41) is the step length, which can be computed using modified nonmonotone line search technique, $h_{k}=\beta^{m_{k}}$, and $m_{k}$ satisfies the following inequalities:

$$
f\left(x_{k}+\beta^{m_{k}} d_{k}\right) \leq f\left(x_{k}\right)+v \beta^{m_{k}}\left(g_{k}^{T} d_{k}+\eta\left\|g_{k}\right\|^{2}\right),
$$

where $v, \beta \in(0,1)$, and $\eta>0$.

\section{Algorithm 4.}

Step 1. Given an initial point $x_{0} \in R^{n}, v \in(0,1), \beta \in(0,1)$, and $\eta>0$; $m$ is a given positive integer, $\rho \in(0,1 / m)$, and $0<\varepsilon \leq 1$, set $k=0$.

Step 2. Compute $g_{k}$; if $\left\|g_{k}\right\| \leq \varepsilon$, then terminate; else go to the next step.

Step 3. Compute $d_{k}$ based on (42).

Step 4. Compute $h_{k}$ based on (44).

Step 5. Set $x_{k+1}=x_{k}+h_{k} d_{k}$

Step 6. Set $k=k+1$ and go to Step 2 .

Lemma 5. For any line search, the search direction $d_{k}$ is defined by (42); then one has

$$
g_{k}^{T} d_{k} \leq(m \rho-1)\left\|g_{k}\right\|^{2}
$$

Proof. If $k \leq m$, so we have

$$
g_{k}^{T} d_{k}=g_{k}^{T}\left(-g_{k}\right)=-\left\|g_{k}\right\|^{2},
$$

and $\rho \in(0,1 / m)$, so it has $0<m \rho<1,-1<m \rho-1<0$.

Hence, we obtain

$$
g_{k}^{T} d_{k}=-\left\|g_{k}\right\|^{2} \leq(m \rho-1)\left\|g_{k}\right\|^{2} .
$$

If $k \geq m+1$, according to (42) and (43), we can obtain the following:

$$
\begin{aligned}
g_{k}^{T} d_{k} & =g_{k}^{T}\left(-g_{k}+\sum_{i=1}^{m} \beta_{k}^{i} d_{k-i}\right) \\
& =-\left\|g_{k}\right\|^{2}+\sum_{i=1}^{m} \frac{\rho\left\|g_{k}\right\|}{\left\|d_{k-i}\right\|} \cdot g_{k}^{T} d_{k-i} \\
& \leq-\left\|g_{k}\right\|^{2}+\sum_{i=1}^{m} \frac{\rho\left\|g_{k}\right\|^{2} \cdot\left\|d_{k-i}\right\|}{\left\|d_{k-i}\right\|} \\
& =-\left\|g_{k}\right\|^{2}+\sum_{i=1}^{m} \rho\left\|g_{k}\right\|^{2}=(m \rho-1)\left\|g_{k}\right\|^{2} .
\end{aligned}
$$

Then, we have

$$
g_{k}^{T} d_{k} \leq(m \rho-1)\left\|g_{k}\right\|^{2}
$$

Hence, (45) is proved.

Lemma 6. For any $k$, then one has

$$
\left\|d_{k}\right\| \leq(1+m \rho)\left\|g_{k}\right\| .
$$

Proof. If $k \leq m$, so we have

$$
\left\|d_{k}\right\|=-\left\|g_{k}\right\| \leq(1+m \rho)\left\|g_{k}\right\| .
$$

If $k \geq m+1$, according to (42) and (43), we can obtain the following:

$$
\begin{aligned}
\left\|d_{k}\right\| & =\left\|-g_{k}+\sum_{i=1}^{m} \beta_{k}^{i} d_{k-i}\right\| \leq\left\|g_{k}\right\|+\sum_{i=1}^{m} \frac{\rho\left\|g_{k}\right\| \cdot\left\|d_{k-i}\right\|}{\left\|d_{k-i}\right\|} \\
& =\left\|g_{k}\right\|+\sum_{i=1}^{m} \rho\left\|g_{k}\right\|=(m \rho-1)\left\|g_{k}\right\| .
\end{aligned}
$$

So we have

$$
\left\|d_{k}\right\| \leq(1+m \rho)\left\|g_{k}\right\|
$$

Hence, (50) is proved.

It is clear that we can draw the following conclusion according to (45) and (50):

$$
\frac{-g_{k}^{T} d_{k}}{\left\|d_{k}\right\|^{2}} \geq \frac{1-m \rho}{(1+m \rho)^{2}} .
$$




\section{The Convergence Properties Analysis for Optimal Algorithm}

In this section, we propose an algorithm and then we study the global convergence property of this algorithm. Firstly, we make the following two assumptions, which have been widely used in the literature to analyze the global convergence of the presented method.

Assumption 7. The objective function $f(x)$ is considered to be bounded in the following circumstances:

$$
\psi=\left\{x \in R^{n} \mid f(x) \leq f\left(x_{0}\right)\right\} .
$$

Assumption 8. The gradient $g(x)$ is Lipschitz continuous; there exists a constant $\xi>0$, for any $x, y \in \psi$; we have

$$
\|g(x)-g(y)\| \leq \xi\|x-y\| .
$$

Lemma 9. Suppose that Assumptions 7 and 8 hold; then Algorithm 4 is well-posed; that is, there exists the step length $h_{k}$, which makes (44) hold.

Proof. According to (44), we have

$$
\begin{aligned}
\lim _{h \rightarrow 0} \frac{f\left(x_{k}+h_{k} d_{k}\right)-f\left(x_{k}\right)-v h_{k}\left(g_{k}^{T} d_{k}+\eta\left\|g_{k}\right\|^{2}\right)}{h_{k}} \\
\quad=g_{k}^{T} d_{k}-v\left(g_{k}^{T} d_{k}+\eta\left\|g_{k}\right\|^{2}\right) \\
\quad=(1-v) g_{k}^{T} d_{k}-v \eta\left\|g_{k}\right\|^{2}<0
\end{aligned}
$$

It indicates that the following formula is set up for when there exists $h_{k}^{\prime}>0$, and $\forall h \in\left[0, h_{k}^{\prime}\right]$.

$$
f\left(x_{k}+h_{k} d_{k}\right)-f\left(x_{k}\right)-v h_{k}\left(g_{k}^{T} d_{k}+\eta\left\|g_{k}\right\|^{2}\right) \leq 0 .
$$

Hence, (44) is proved.

Lemma 10. Suppose that Assumptions 7 and 8 hold, $\eta \in(0,1-$ $\mathrm{m} \rho$ ), and there is a constant $\delta$; then

$$
f\left(x_{k}\right)-f\left(x_{k+1}\right) \geq \delta\left\|g_{k}\right\|^{2} .
$$

Proof. $\bar{h}=\inf _{\forall k}\left\{h_{k}\right\}$ is defined; we just need proof that $\bar{h}>0$. First of all, we prove it with a contradiction; suppose that $\bar{h}=$ 0 holds, and there are a series of subfamily $\left\{h_{k}\right\}$; then

$$
\lim _{k \rightarrow+\infty} h_{k}=0
$$

Set $h=h_{k} / \beta$, and $h_{k}$ is the maximum in $\left\{1, \beta, \beta^{2}, \cdots\right\}$, which meets (44). Thus, $h=h_{k} / \beta>h_{k}$ can not meet (44). Then

$$
f\left(x_{k}+h d_{k}\right)>f\left(x_{k}\right)+v h\left(g_{k}^{T} d_{k}+\eta\left\|g_{k}\right\|^{2}\right) .
$$

Further, we have

$$
f\left(x_{k}+h d_{k}\right)>f\left(x_{k}\right)+v h g_{k}^{T} d_{k}
$$

Based on the mean value theorem, then

$$
f\left(x_{k}+h d_{k}\right)-f\left(x_{k}\right)=g\left(x_{k}+h \zeta_{k} d_{k}\right)^{T} \cdot h d_{k},
$$

in which $\zeta_{k} \in[0,1]$.

Thus, it follows from (62) and (63) that

$$
g\left(x_{k}+h \zeta_{k} d_{k}\right)^{T} d_{k}<v g_{k}^{T} d_{k}
$$

From Assumption 8 and Cauchy Schwartz inequality, we can obtain

$$
\begin{aligned}
& {\left[g\left(x_{k}+h \zeta_{k} d_{k}\right)-g_{k}\right]^{T} d_{k}} \\
& \quad \leq\left\|g\left(x_{k}+h \zeta_{k} d_{k}\right)-g_{k}\right\| \cdot\left\|d_{k}\right\| \\
& \quad \leq \xi \cdot \zeta_{k} \cdot h\left\|d_{k}\right\| \cdot\left\|d_{k}\right\| \leq \xi h\left\|d_{k}\right\|^{2} .
\end{aligned}
$$

From (64) and (65), it follows that

$$
(v-1) g_{k}^{T} d_{k}<\left[g\left(x_{k}+h \zeta_{k} d_{k}\right)-g_{k}\right]^{2} \leq \xi h\left\|d_{k}\right\|^{2} .
$$

Then, we have

$$
h \geq \frac{1-v}{\xi}\left(\frac{-g_{k}^{T} d_{k}}{\left\|d_{k}\right\|^{2}}\right) .
$$

Combining (67) and (54), we obtain

$$
h_{k}=h \beta \geq \frac{\beta(1-v)}{\xi} \cdot \frac{(1-m \rho)}{(1+m \rho)^{2}}>0 .
$$

Obviously, we clearly see that (68) and (60) are contradictory. Hence, we have

$$
\bar{h}>0 \text {. }
$$

Then, combining (44) and (45), we can obtain

$$
\begin{aligned}
f\left(x_{k}\right)-f\left(x_{k+1}\right) & \geq-v h_{k}\left(g_{k}^{T} d_{k}+\eta\left\|g_{k}\right\|^{2}\right) \\
& \geq v h_{k}\left[(1-m \rho)\left\|g_{k}\right\|^{2}-\eta\left\|g_{k}\right\|^{2}\right] \\
& \geq v \bar{h}(1-m \rho-\eta)\left\|g_{k}\right\|^{2},
\end{aligned}
$$

in which, set $\delta=v \bar{h}(1-m \rho-\eta)$.

Hence, (59) is proved.

According to $\lim _{k \longrightarrow \infty}$ inf $\left\|g_{k}\right\|=0$ (see $[50,51]$ ), it is clear that the sequence $\left\{\mathrm{x}_{\mathrm{k}}\right\}$ is contained in $\psi$, and there exists a constant $f^{*}$, such that

$$
\lim _{k \rightarrow \infty} f\left(\mathrm{x}_{\mathrm{k}}\right)=f^{*} .
$$

$\forall \mathrm{x}, \mathrm{y} \in[\mathrm{a}, \mathrm{b}]$, suppose $\mathrm{a}<\mathrm{x}<\mathrm{y}<\mathrm{b}$; then it can satisfy the following conditions: $\mathrm{a}+\Delta \mathrm{x}<\mathrm{x}, \mathrm{b}-\Delta \mathrm{x}\rangle \mathrm{y}$ for $\Delta \mathrm{x}$ small enough. Because $f(\mathrm{x})$ is a convex function, so we obtain

$$
\begin{aligned}
\frac{f(\mathrm{a}+\Delta \mathrm{x})-f(\mathrm{a})}{\Delta \mathrm{x}} & \leq \frac{f(\mathrm{y})-f(\mathrm{x})}{\mathrm{y}-\mathrm{x}} \\
& \leq \frac{f(\mathrm{~b})-f(\mathrm{~b}-\Delta \mathrm{x})}{\triangle \mathrm{x}} .
\end{aligned}
$$


Let $\triangle \mathrm{x} \longrightarrow 0^{+}$; we have

$$
f_{+}^{\prime}(\mathrm{a}) \leq \frac{f(\mathrm{y})-f(\mathrm{x})}{\mathrm{y}-\mathrm{x}} \leq f_{-}^{\prime}(\mathrm{b}) .
$$

Set $\mathrm{K}=\max \left\{\left|f_{+}^{\prime}(\mathrm{a})\right|,\left|f_{-}^{\prime}(\mathrm{b})\right|\right\}$, then

$$
\left|\frac{f(\mathrm{y})-f(\mathrm{x})}{\mathrm{y}-\mathrm{x}}\right| \leq \mathrm{K} .
$$

Hence, we get

$$
|f(\mathrm{x})-f(\mathrm{y})| \leq \mathrm{K}|\mathrm{x}-\mathrm{y}| \text {. }
$$

Therefore, according to the above discussion, we can draw a conclusion that the objective function $f$ defined in (15) is differentiable and Lipschitz continuous.

\section{Examples and Discussions}

In this section, a mathematical example and an engineering example are investigated to clarify how to solve the solution of the ill-posed problem quickly and accurately by using a novel fractional Tikhonov regularization (NFTR) coupled with an improved super-memory gradient (ISMG) method.

6.1. A Mathematical Example. In this section, our first example is a severely ill-posed Fredholm integral equation of the first kind given by

$$
\int_{0}^{\pi} \exp (\tau \cos (t)) x(t) \mathrm{d} t=2 \frac{\sinh (\tau)}{\tau} \quad 0 \leq \tau \leq \frac{\pi}{2} .
$$

Set $\mathrm{C}(t, \tau)=\exp (\tau \cos (t)), \mathrm{y}(\tau)=2(\sinh (\tau) / \tau)$; then

$$
\int_{0}^{\pi} \mathrm{C}(t, \tau) \mathrm{x}(t) \mathrm{d} t=\mathrm{y}(\tau) \quad 0 \leq \tau \leq \frac{\pi}{2}
$$

in which the perturbation of the right-hand side is set as follows:

$$
\mathrm{y}(\tau)=\widehat{\mathrm{y}}(\tau)+e
$$

where $e=\delta \cdot(y(\tau)) \cdot$ rand, in which $\operatorname{std}(\mathrm{y}(\tau))$, rand, and $\delta$ denote the standard deviation of $\mathrm{y}(\tau)$, a random number within $[-1,1]$, and the noise level, respectively.

Then (77) was discretized, which can be obtained:

$$
\frac{\pi}{n} \sum_{\mathrm{i}=0}^{\mathrm{n}} \mathrm{C}\left(t_{i}, \tau_{k}\right) \mathrm{x}\left(t_{i}\right)=\mathrm{y}\left(\tau_{k}\right) \quad k=0,1, \ldots n,
$$

in which $t_{i}=\pi i / n, \tau_{k}=\pi k / 2$.

The problem of solving (76) is an ill-posed problem; we solve the approximate solution of (76) with the right-hand side given a perturbation. The true solution of $(76)$ is $\mathrm{x}(t)=$ $\sin (t)$; then we need to calculate the identified solution $\widehat{x}(t)$. Here, TR method, FTR method, and the present method in this paper are applied to deal with the problem.

In order to further discuss the quality of the different methods used and assess the degree of the approximation of the identified result to the true result, we provide the following evaluation metrics.

Relative error (RE):

$$
\mathrm{RE}=\frac{\|\widehat{\mathrm{x}}-\mathrm{x}\|}{\|\mathrm{x}\|} .
$$

Correlation coefficient (CC):

$$
\mathrm{CC}=\frac{\sum_{i=0}^{n}[\widehat{\mathrm{x}}-\mathrm{E}(\widehat{\mathrm{x}})][\mathrm{x}-\mathrm{E}(\mathrm{x})]}{\|\widehat{\mathrm{x}}-\mathrm{E}(\widehat{\mathrm{x}})\| \cdot\|\mathrm{x}-\mathrm{E}(\mathrm{x})\|} .
$$

The program processing is executed in the MATLAB regularization tools package, and the condition number of the matrix $\mathbf{C}$ is calculated by the MATLAB function cond; it can be found that cond $(C)=2 \cdot 10^{37}$, which means that the matrix is singular.

In our experiments, the noise vector $e$ is selected as a normal random distribution vector with zero mean and normalized to an ideal noise level:

$$
\triangle=\frac{\|e\|_{2}}{\|y\|_{2}} .
$$

In this experiment, the regularization parameter $\mu$ is selected by using the discrepancy principle, in which $\|e\|_{2}=\delta=\ell$ and $\omega=1.01$. Other parameters are set to the following: $v=0.5$, $\beta=0.3, \eta=0.2, m=2$, and $\varepsilon=10^{-3}$. The computer stops the iteration processing when $\left|g_{k}\right| \leq 10^{-3}$ is met.

$\alpha^{*}$ denotes the optimal value of the fractional order $\alpha \epsilon$ $\{0,0.1,0.2, \ldots 1.0\}$ that gives the most accurate identified result of (76) when $\mu$ is selected using the discrepancy principle $\left(\|e\|_{2}=\delta=\ell\right.$ and $\left.\omega=1.01\right)$.

In this section, we employ the standard TR, FTR, and the present method in the paper to identify the results of $x(t)$, respectively.

First of all, when $n \in[20,30,40,50]$ and the noise level is set to $10 \%, 5 \%$, and $1 \%$, then RE and CC and iterative steps of the identified result are listed in Table 1.

Table 1 shows that whether the noise level is set to $10 \%$, $5 \%$, or $1 \%$, RE and iterative steps of the identified result with application of the above methods are seen to increase with $n$; then CC is the opposite.

Moreover, Table 1 also shows that when $n \in$ $[20,30,40,50], \mathrm{RE}$ and iterative steps of the identified result with application of the above methods are seen to increase with the noise level; then CC is the opposite.

It can be seen clearly that the evaluation indicators using the present method are associated with minimum RE, minimum iterative steps, and maximum CC in comparison to the standard TR method and FTR method.

In a word, Table 1 shows that the identified result of the present method in this paper is closer to the true solution than that of the other methods.

Hence, it can be seen clearly that the evaluation indicators using the present method are associated with minimum RE, minimum iterative steps, and maximum CC in comparison to the other methods, which demonstrates that the present method in this paper is superior to the standard TR and FTR method. 
TABLE 1: The evaluation metrics comparisons of different methods.

\begin{tabular}{|c|c|c|c|c|c|}
\hline$n$ & Noise level (\%) & Methods & $\mathrm{RE}$ & $\mathrm{CC}$ & Iterative steps \\
\hline \multirow{9}{*}{20} & 10 & \multirow{3}{*}{ The standard TR } & 0.1136 & 0.8950 & 33 \\
\hline & 5 & & 0.1128 & 0.8960 & 30 \\
\hline & 1 & & 0.1109 & 0.8966 & 27 \\
\hline & 10 & \multirow{3}{*}{ FTR } & 0.1080 & 0.8994 & 25 \\
\hline & 5 & & 0.1069 & 0.9218 & 22 \\
\hline & 1 & & 0.1052 & 0.9268 & 20 \\
\hline & 10 & \multirow{3}{*}{ Present } & 0.0483 & 0.9858 & 12 \\
\hline & 5 & & 0.0368 & 0.9876 & 10 \\
\hline & 1 & & 0.0224 & 0.9882 & 7 \\
\hline \multirow{9}{*}{30} & 10 & \multirow{3}{*}{ The standard TR } & 0.1183 & 0.8946 & 40 \\
\hline & 5 & & 0.1180 & 0.8958 & 38 \\
\hline & 1 & & 0.1179 & 0.8964 & 33 \\
\hline & 10 & \multirow{3}{*}{ FTR } & 0.1177 & 0.8969 & 30 \\
\hline & 5 & & 0.1163 & 0.9200 & 26 \\
\hline & 1 & & 0.1111 & 0.9213 & 22 \\
\hline & 10 & \multirow{3}{*}{ Present } & 0.0591 & 0.9709 & 16 \\
\hline & 5 & & 0.0503 & 0.9712 & 12 \\
\hline & 1 & & 0.0407 & 0.9732 & 11 \\
\hline \multirow{9}{*}{40} & 10 & \multirow{3}{*}{ The standard TR } & 0.1199 & 0.8870 & 48 \\
\hline & 5 & & 0.1196 & 0.8876 & 46 \\
\hline & 1 & & 0.1187 & 0.8881 & 43 \\
\hline & 10 & \multirow{3}{*}{ FTR } & 0.1184 & 0.8889 & 38 \\
\hline & 5 & & 0.1182 & 0.8894 & 32 \\
\hline & 1 & & 0.1174 & 0.8899 & 27 \\
\hline & 10 & \multirow{3}{*}{ Present } & 0.0699 & 0.9678 & 18 \\
\hline & 5 & & 0.0653 & 0.9695 & 13 \\
\hline & 1 & & 0.0618 & 0.9603 & 12 \\
\hline \multirow{9}{*}{50} & 10 & \multirow{3}{*}{ The standard TR } & 0.1219 & 0.8776 & 52 \\
\hline & 5 & & 0.1214 & 0.8780 & 50 \\
\hline & 1 & & 0.1203 & 0.8787 & 47 \\
\hline & 10 & \multirow{3}{*}{ FTR } & 0.1198 & 0.8790 & 44 \\
\hline & 5 & & 0.1188 & 0.8799 & 34 \\
\hline & 1 & & 0.1144 & 0.8799 & 30 \\
\hline & 10 & \multirow{3}{*}{ Present } & 0.0706 & 0.9012 & 20 \\
\hline & 5 & & 0.0701 & 0.9093 & 15 \\
\hline & 1 & & 0.0681 & 0.9117 & 13 \\
\hline
\end{tabular}

6.2. An Engineering Example. In this section, the present method is applied to an engineering example of the identification problem of dynamic force generated between conical pick and coal-seam structure.

6.2.1. Material Properties. A schematic of conical pick and coal-seam structure is shown in Figure 1. In this diagram, the size of the coal-seam structure specimen manufactured artificially was set as $2000 \mathrm{~mm} \times 800 \mathrm{~mm} \times 1750 \mathrm{~mm}$. The density of conical pick is $7800 \mathrm{~kg} / \mathrm{m}^{3}$ and the density of coal-seam structure is $1607.98 \mathrm{~kg} / \mathrm{m}^{3}$; the elastic modulus of conical pick is $600 \mathrm{MPa}$ and the elastic modulus of coal-seam structure is $322.09 \mathrm{MPa}$; the Poisson ratio of conical pick is 0.25 and the Poisson ratio of coal-seam structure is 0.25 , respectively [52-54].
6.2.2. Experimental Procedure. In our current study, the experimental procedure was carried out on an experimental setup with rotatable and adjustable parameter which was shown in Figure 2. The experimental setup includes a test bed and a test system. The test bed mainly includes the following devices: motor, reducer, coupling, cutting mechanism, platform, and coal-seam structure. The test system includes the force-measured device, force sensor, the signal amplifier, and the DaspV10 intelligent data acquisition and signal processing system [55].

It is clearly seen from Figure 3 that the conical pick is installed on the force-measured device, the various surfaces of which are intercepting with the force sensor, once the coal-seam structure is hit by conical pick, the deformation of a force sensor is converted into a signal, which is called 


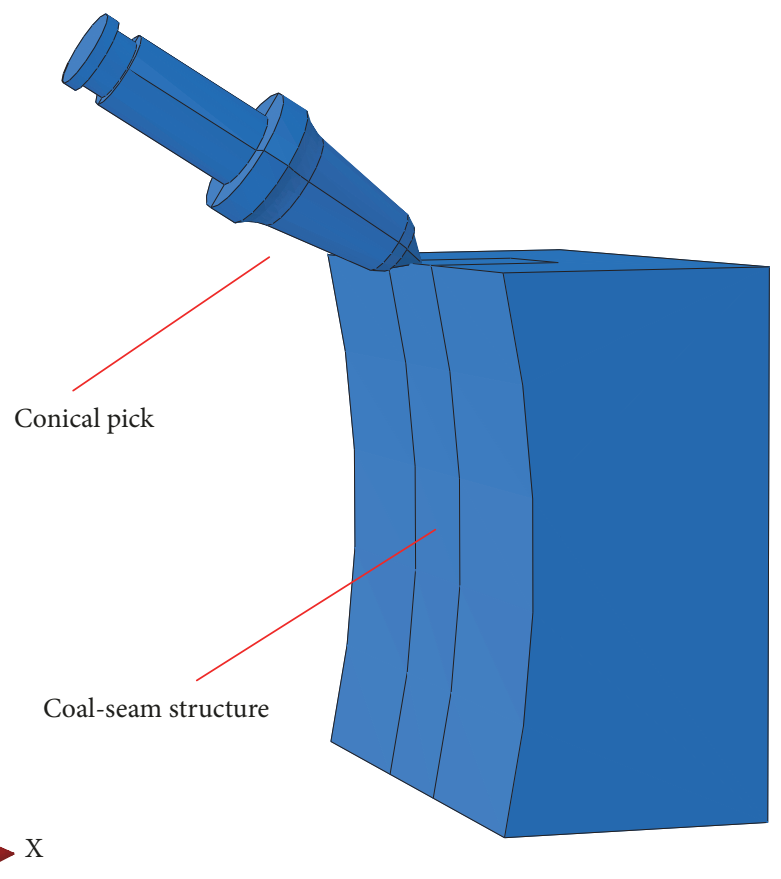

FIgURE 1: A schematic of conical pick and coal-seam structure.

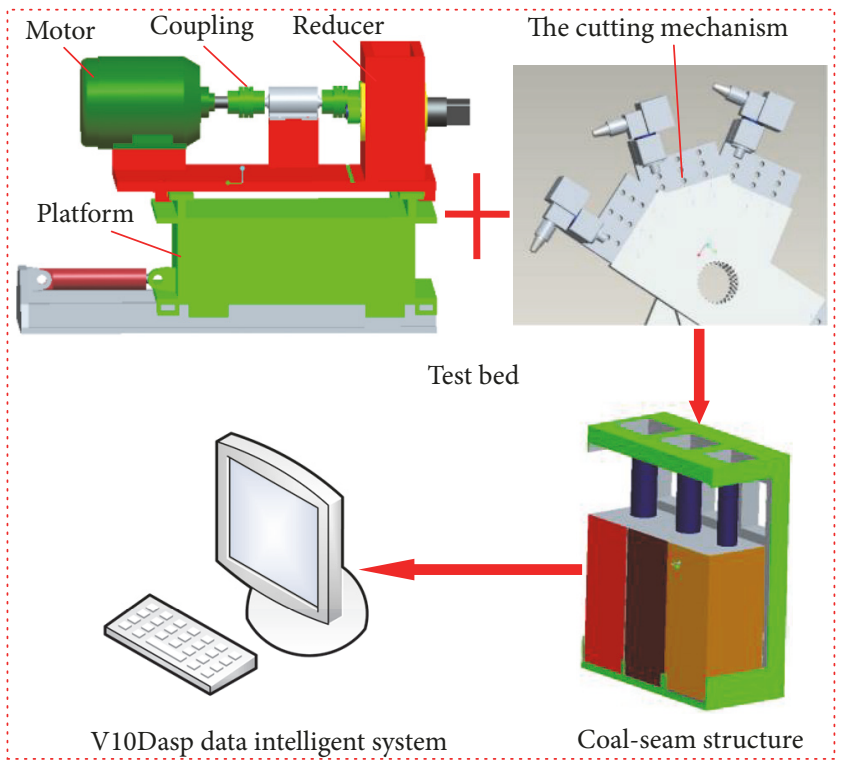

FIGURE 2: Experimental procedure.

the dynamic force, then the dynamic force is converted by a signal amplifier, finally recorded by V10Dasp data intelligent acquisition and signal processing system.

6.2.3. The Selection of the Optimal Fractional Order. In our experiment, the installation angle of conical pick is $45^{\circ}$, the maximum cutting thickness of conical pick is $20 \mathrm{~mm}$, the hauling speed is $0.8 \mathrm{r} / \mathrm{min}$, and the rotary arm speed is $30 \mathrm{~m} / \mathrm{min}$. In order to effectively identify dynamic force generated between conical pick and coal-seam structure, first

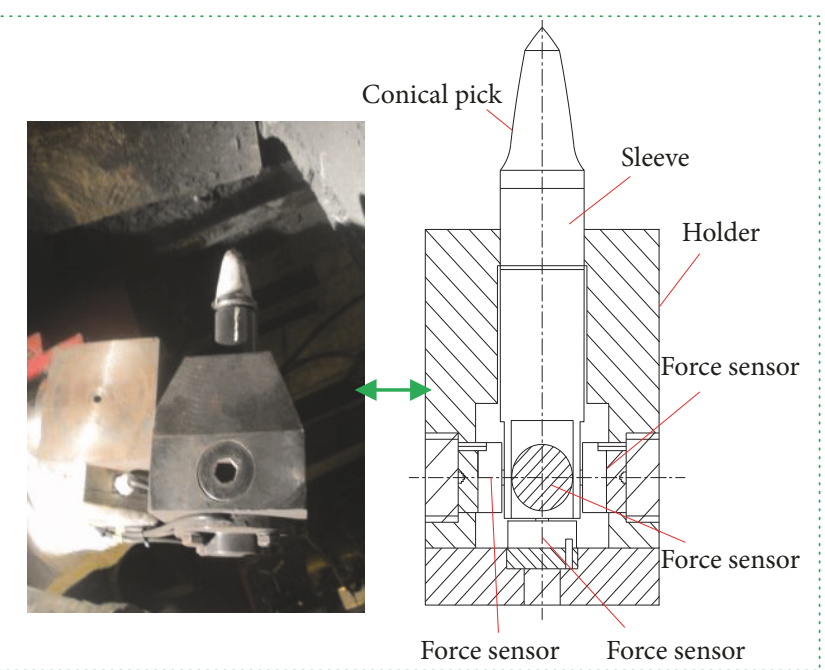

FIGURE 3: The dynamic force-measured device.

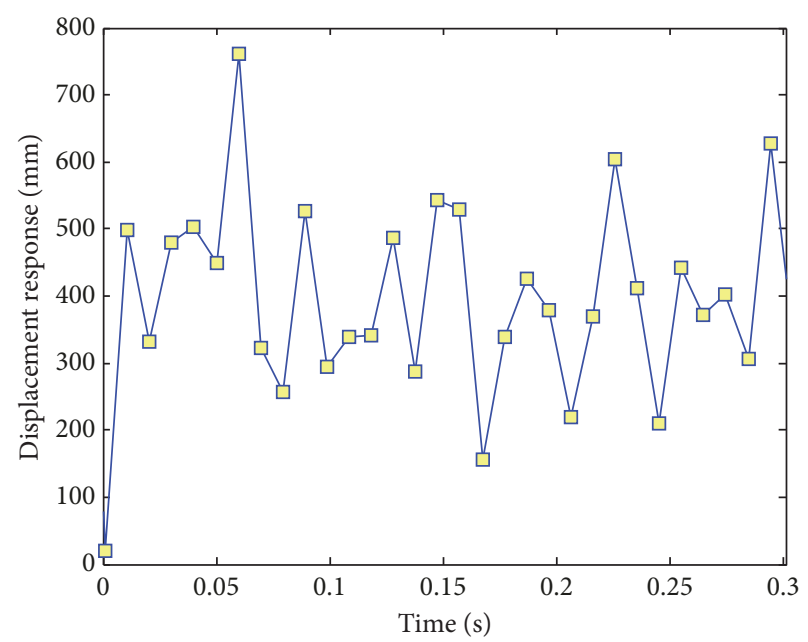

Figure 4: The measured displacement response.

we provide the measured displacement response within 0.3 seconds, which is shown in Figure 4. Since the measured displacement response can be given by the experiment, then we can use the present method in the paper to identify dynamic force.

In this part, we can study the effect of different fractional order on the results of dynamic force identification in order to determine the most optimal fractional order.

First of all, all program codes were made in MATLAB 7.0 and Windows 7 operating system. On the one hand, $d_{k}$ is obtained using (42); all parameters are set to the following: $\omega=1.01, v=0.5, \beta=0.3, \eta=0.2, m=2$, and $\varepsilon=10^{-3}$. The iteration processing stops when $\left|g_{k}\right| \leq 10^{-3}$ is met.

The identified results of dynamic force are achieved by using the present method in this paper, which are shown in Figures 5(a), 5(b), 5(c), 5(d), 5(e), 5(f), 5(g), 5(h), and 5(i), respectively. RE and $\mathrm{CC}$ and iterative steps of the identified results under different fractional order $(\alpha)$ are listed in Table 2. 


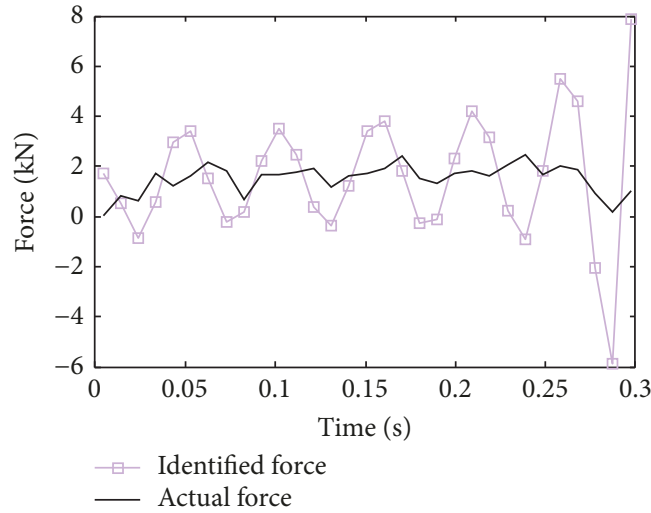

(a) $\alpha=0.1$

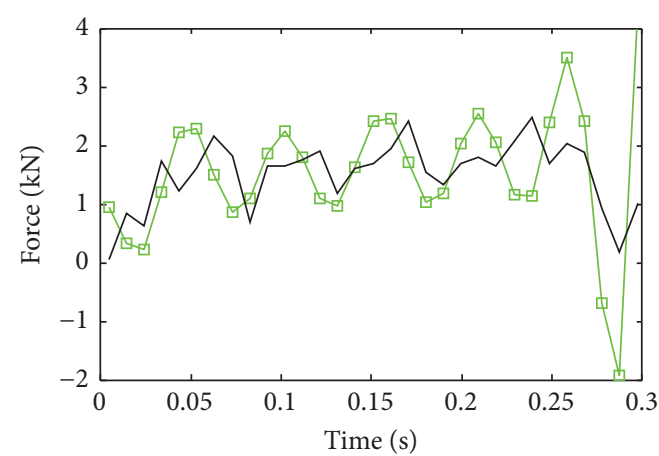

$\square$ Identified force

- Actual force

(c) $\alpha=0.3$

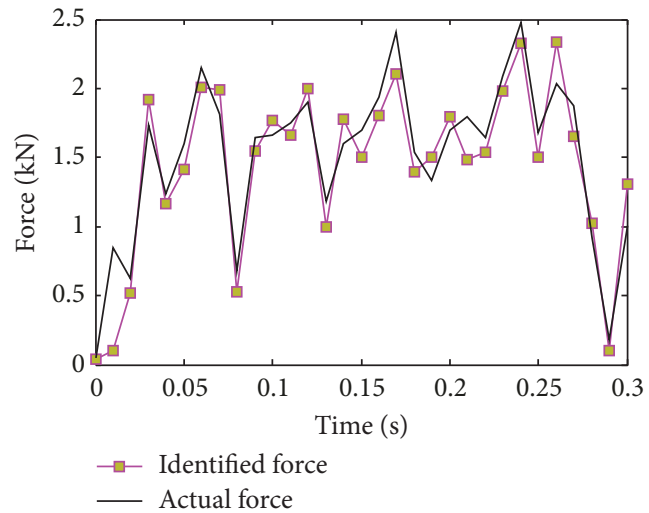

(e) $\alpha=0.5$

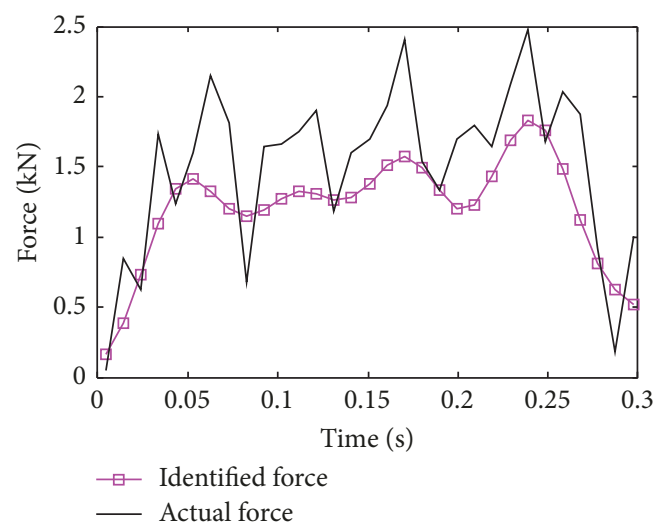

(g) $\alpha=0.7$

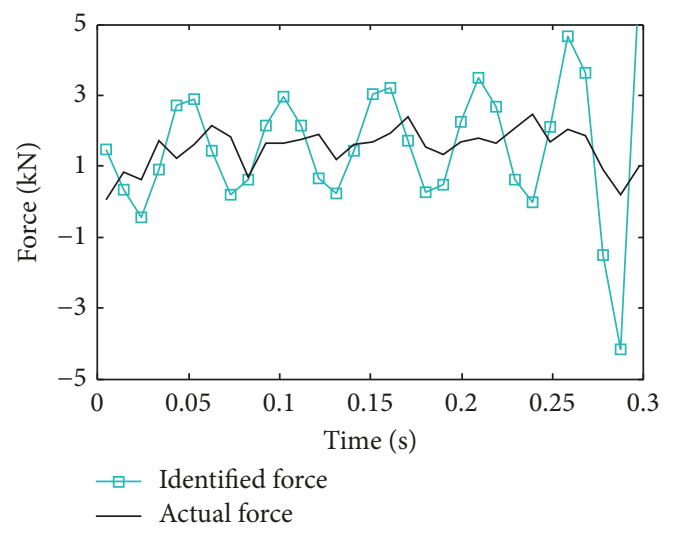

(b) $\alpha=0.2$

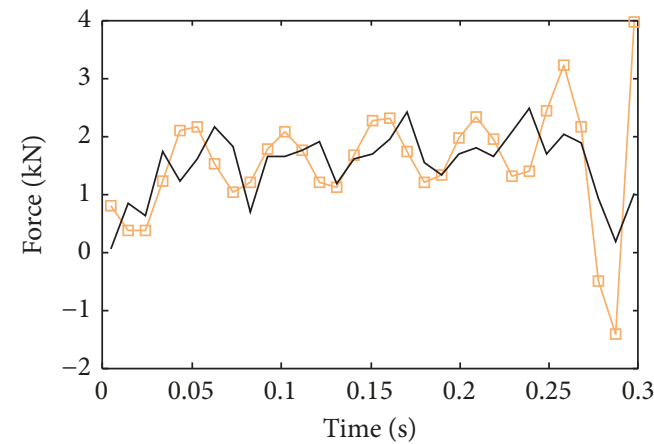

- Identified force

Actual force

(d) $\alpha=0.4$

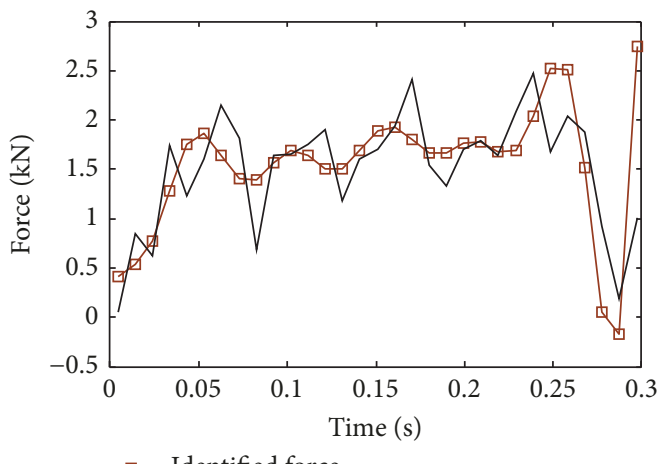

$\square-$ Identified force

- Actual force

(f) $\alpha=0.6$

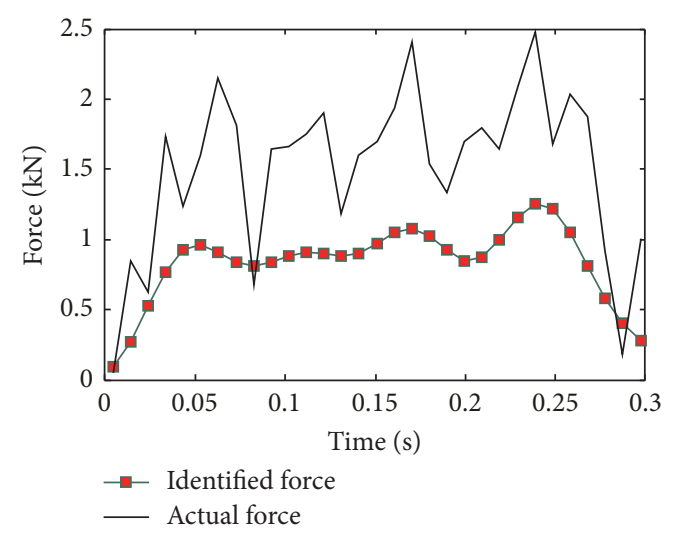

(h) $\alpha=0.8$

FIgURE 5: Continued. 


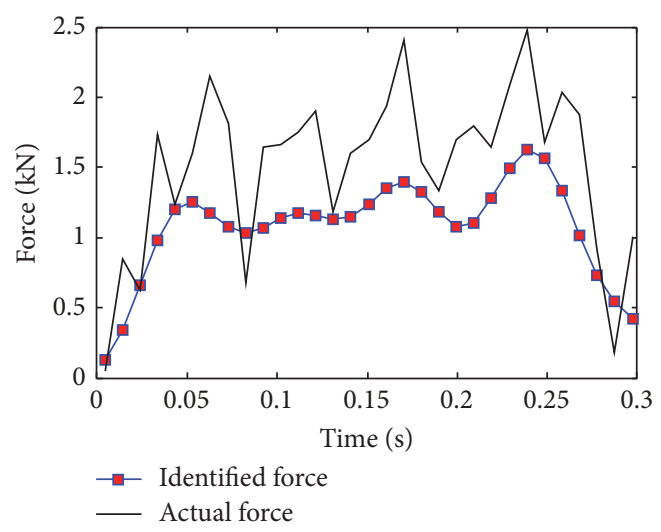

(i) $\alpha=0.9$

FIgURE 5: The identified results under different fractional order.

TABLE 2: The selection of the optimal fractional order.

\begin{tabular}{lccccccccc}
\hline \multirow{2}{*}{ Evaluation metrics } & \multicolumn{9}{c}{ Fractional order $\alpha$} \\
& 0.1 & 0.2 & 0.3 & 0.4 & 0.5 & 0.6 & 0.7 & 0.8 & 0.9 \\
\hline $\mathrm{RE}$ & 1.4687 & 1.1069 & 0.6304 & 0.5295 & 0.1038 & 0.3119 & 0.2825 & 0.4811 & 0.3463 \\
$\mathrm{CC}$ & 0.3410 & 0.3686 & 0.4649 & 0.5058 & 0.9422 & 0.6583 & 0.8200 & 0.8188 & 0.8199 \\
Iterative steps & 60 & 53 & 47 & 44 & 9 & 29 & 22 & 39 & 32 \\
\hline
\end{tabular}

Figure 5(a) illustrates that the identified force is gained at $\alpha=0.1$, and Figure 5(b) provides the identified force at $\alpha=0.2$, the identified force at $\alpha=0.3$ can be seen from Figure 5(c), Figure 5(d) gives the identified force at $\alpha=0.4$, the identified force at $\alpha=0.5$ is shown in Figure 5(e), Figure 5(f) illustrates that the identified force is gained at $\alpha=0.6$, Figure $5(\mathrm{~g})$ provides the identified force at $\alpha=0.7$, the identified force at $\alpha=0.8$ can be seen from Figure 5(h), and Figure 5(i) gives the identified force at $\alpha=0.9$

We can see from Figures 5(a), 5(b), 5(c), 5(d), 5(e), 5(f), $5(\mathrm{~g}), 5(\mathrm{~h})$, and $5(\mathrm{i})$ that the different fractional order can identify the dynamic force generated between conical pick and coal-seam structure from the measured displacement response. However, the performance of dynamic force identification results has a certain difference.

Figures 5(a) and 5(b) illustrate that the identified force is not ideal owing to the smaller fractional order. On the contrary, when the fractional order is larger, the identified force is very seriously deviating from the actual force, which can be seen from Figures 5(h) and 5(i). Although Figures 5(c) and 5(d) can identify the contours of the load, there is a big error with the actual load. Also, Figures 5(f) and 5(g) can also identify the contours of the load, but not the ideal solution we want. However, we can find that Figure 5(e) is the relatively ideal result that we expect. Hence, within the scale of experiment, we know that the optimal fractional order of dynamic force identification using the present method is 0.5 .

In order to confirm the optimal fractional order, RE and CC and iterative steps are listed in Table 2. It is clearly seen from Table 2 that RE and iterative steps of Figures 5(a), 5(b), 5(c), and 5(d) are larger than those of Figure 5(e). However, $\mathrm{RE}$ and iterative steps of Figure 5(e) are smaller than those of Figures 5(f), 5(g), 5(h), and 5(i). And the CC value is the opposite. Hence, compared with the other fractional order, we find from Table 2 that the fractional order $\alpha=0.5$ has the smallest RE, the largest CC, and the least iterative steps.

Taking into account the above analysis, we can draw a conclusion that the optimal fractional order $(\alpha=0.5)$ using the present method makes it more successful to identify dynamic force generated between conical pick and coal-seam structure based on the measured displacement response.

6.2.4. Comparison of Dynamic Force Identification Methods. The proposed method in this paper is compared with the standard TR method and FTR method, and the identified results of the three methods are shown in Figures 6(a), 6(b), and $6(\mathrm{c})$, respectively.

Figure 6(a) explains that the identified force is gained using the standard TR method at $\alpha=1.0$, Figure 6(b) explains that the identified force is given by FTR method at $\alpha^{*}=0.4$, and the identified result at $\alpha^{*}=0.5$ is shown in Figure 6(c) using the present method in the paper.

We can find from Figures 6(a), 6(b), and 6(c) that the three methods can all identify dynamic force; however, the present method in the paper can successfully and effectively identify the details of dynamic force in comparison to the standard TR method and FTR method.

In order to deeply distinguish the stability of the proposed method in this paper, we give the filter functions curves of the three methods with their corresponding optimal regular parameters $\left(\mu=10^{-2}\right)$ shown in Figure 7 . In addition, the evaluation metrics of the identified results through three methods are listed in Table 3.

We can clearly find from Table 3 that the present method under the fractional order $\alpha^{*}=0.5$ has the smallest RE, the largest CC, and the least iterative steps. 
TABLE 3: Comparison of different identification methods.

\begin{tabular}{lccc}
\hline Evaluation metrics & The standard TR method $(\alpha=1.0)$ & FTR method $\left(\alpha^{*}=0.4\right)$ & Present method $\left(\alpha^{*}=0.5\right)$ \\
\hline RE & 0.2673 & 0.2033 & 0.1038 \\
CC & 0.7098 & 0.7799 & 0.9422 \\
Iterative steps & 22 & 15 & 9 \\
\hline
\end{tabular}

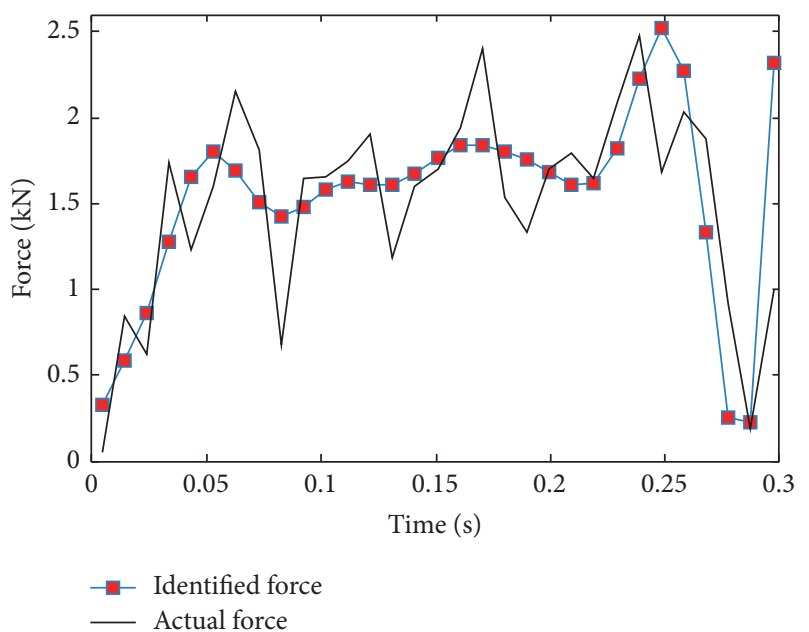

(a) The standard TR method

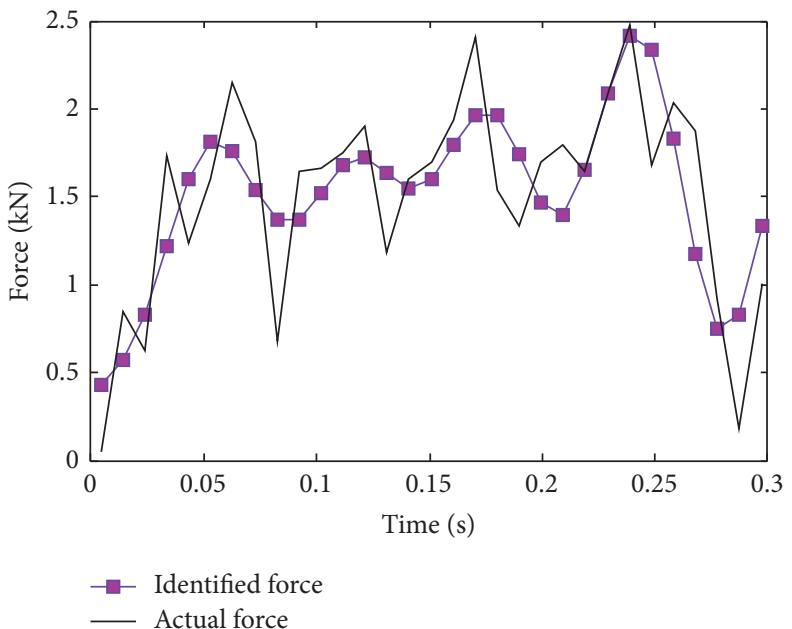

(b) FTR method

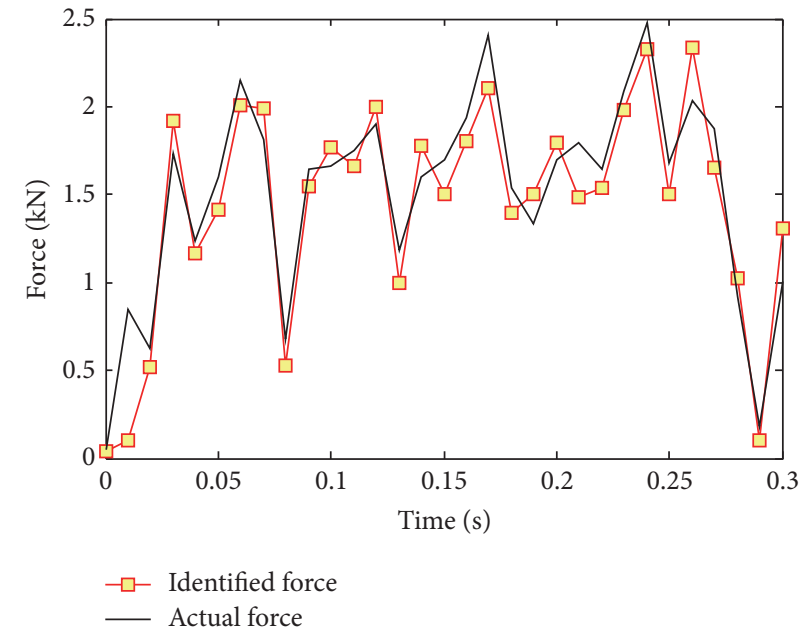

(c) Present method

FIGURE 6: The comparison of identified results under different methods.

Figure 7 shows that the three curves basically coincided in the small singular value part with the regular parameters. Therefore, the three methods can be used to modify small singular values to suppress uncertain noise. However, with regard to the parts of the middle and large singular value, the three filter functions $\Omega\left(\alpha, \sigma_{i}\right)$ of the present method in the paper have relatively larger value in comparison to the standard TR and FTR method. In addition, Table 3 demonstrates that the identified result of the proposed method is better than that of the standard TR method and FTR method.

Taking into account the above analysis and discussion, we can conclude that the filter functions $\Omega\left(\alpha, \sigma_{i}\right)$ of the present method in the paper can not only retain the details of the solution components corresponding to smaller singular values, but also resist the solution components corresponding to larger singular values. In other words, the present method in the paper can reduce the ill-posedness of dynamic force identification and can offer a stable identified result.

\section{Conclusions}

This paper presents a novel inverse technique to offer an optimal solution for the ill-posed problems in mathematical and practical engineering. The inverse problem models are uniformly represented as a Fredholm integral equation of the first kind which has the ill-posedness to a certain extent. A novel fractional Tikhonov regularization (NFTR) method is proposed to perform an effective inverse identification. 


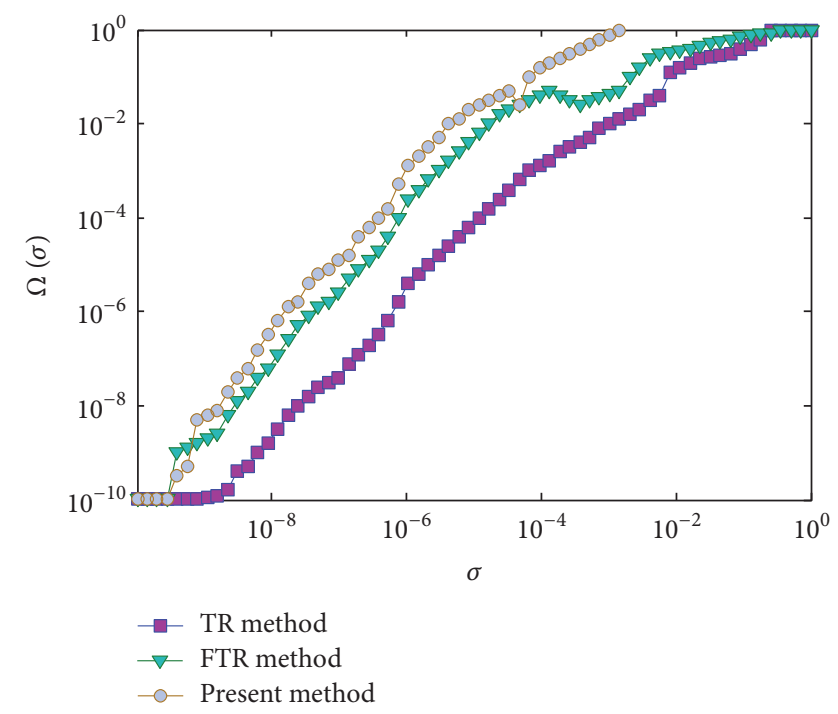

FIGURE 7: The curve of the filter factor with the singular value under different methods.

The smoothing functional of the ill-posed problem processed by the proposed method is considered as an unconstrained optimization problem; an improved super-memory gradient (ISMG) method is introduced to offer an optimal solution.

A mathematical problem and an engineering example of the identification problem of dynamic force generated between conical pick and coal-seam structure show that the result of the present method is compared with that of the standard TR and FTR methods; new findings can be seen; that is, the present method has a better effect and stronger robustness, which can not only improve accuracy and stability of the inverse identification problem, but also accelerate the rate of convergence and enhance the details. Therefore, we can make a clear conclusion that the present method in this paper can reduce the ill-posedness of the inverse problems and provide a unified optimization solution. In addition, there is reason to believe that the present method can offer a reference for future research.

\section{Data Availability}

This section describes the data used in this research. Readers can find the source of data in the following ways: (i) https://www.hindawi.com/journals/mpe/2017/9125734/ and (ii) https://doi.org/10.4028/www.scientific.net/AMM.577.196.

\section{Conflicts of Interest}

The authors declare that they have no conflicts of interest regarding the publication of this article.

\section{Acknowledgments}

This work was supported by the Chinese National Natural Science Foundation (Contracts nos. 51674106 and 51274091).

\section{References}

[1] T. S. Jang, H. Baek, S. L. Hana, and T. Kinoshita, "Indirect measurement of the impulsive load to a nonlinear system from dynamic responses: inverse problem formulation," Mechanical Systems and Signal Processing, vol. 24, no. 6, pp. 1665-1681, 2010.

[2] T. Hein and B. Hofmann, "On the nature of ill-posedness of an inverse problem arising in option pricing," Inverse Problems, vol. 19, no. 6, pp. 1319-1338, 2003.

[3] T. S. Jang, H. Baek, M. C. Kim, and B. Y. Moon, "A new method for detecting the time-varying nonlinear damping in nonlinear oscillation systems: nonparametric identification," Mathematical Problems in Engineering, Art. ID 749309, 12 pages, 2011.

[4] S.-J. Kim and S.-K. Lee, "Experimental identification for inverse problem of a mechanical system with a non-minimum phase based on singular value decomposition," Journal of Mechanical Science and Technology, vol. 22, no. 8, pp. 1504-1509, 2008.

[5] N. S. Hoang and A. G. Ramm, "Solving ill-conditioned linear algebraic systems by the dynamical systems method," Inverse Problems in Science and Engineering, vol. 16, no. 5, pp. 617-630, 2008.

[6] W. Luo, "Parameter identifiability of ship manoeuvring modeling using system identification," Mathematical Problems in Engineering, vol. 2016, Article ID 8909170, 10 pages, 2016.

[7] A. Beck and A. Ben-Tal, "On the solution of the Tikhonov regularization of the total least squares problem," SIAM Journal on Optimization, vol. 17, no. 1, pp. 98-118, 2006.

[8] H. Egger and H. W. Engl, “Tikhonov regularization applied to the inverse problem of option pricing: convergence analysis and rates," Inverse Problems, vol. 21, no. 3, pp. 1027-1045, 2005.

[9] S. G. Solodky and G. L. Myleiko, "About regularization of severely ill-posed problems by standard Tikhonov's method with the balancing principle," Mathematical Modelling and Analysis, vol. 19, no. 2, pp. 199-215, 2014.

[10] S. George and P. Jidesh, "Reconstruction of signals by standard Tikhonov method," Applied Mathematical Sciences, vol. 5, no. 57-60, pp. 2819-2829, 2011.

[11] T. S. Jang, H. G. Sung, S. L. Han, and S. H. Kwon, "Inverse determination of the loading source of the infinite beam on elastic foundation," Journal of Mechanical Science \& Technology, vol. 22, no. 12, pp. 2350-2356, 2008.

[12] J. Gao, D. Wang, and J. Peng, "A Tikhonov-type regularization method for identifying the unknown source in the modified Helmholtz equation," Mathematical Problems in Engineering, vol. 2012, Article ID 878109, 13 pages, 2012.

[13] V. Melicher and V. Vrabel, "On a continuation approach in Tikhonov regularization and its application in piecewiseconstant parameter identification," Inverse Problems, vol. 29, no. 11, pp. 193-212, 2013.

[14] B. Weber, P. Paultre, and J. Proulx, "Structural damage detection using nonlinear parameter identification with Tikhonov regularization," Structural Control and Health Monitoring, vol. 14, no. 3, pp. 406-427, 2007.

[15] V. A. Postnov, "Use of Tikhonov's regularization method for solving identification problem for elastic systems," Mechanics of Solids, vol. 45, no. 1, pp. 51-56, 2010.

[16] T. A. Johansen, "On Tikhonov regularization, bias and variance in nonlinear system identification," Automatica, vol. 33, no. 3, pp. 441-446, 1997. 
[17] D. Gerth, E. Klann, R. Ramlau, and L. Reichel, "On fractional Tikhonov regularization," Journal of Inverse and ILL-Posed Problems, vol. 23, no. 6, pp. 611-625, 2015.

[18] T. K. Huckle and M. Sedlacek, "Tikhonov-Phillips regularization with operator dependent seminorms," Numerical Algorithms, vol. 60, no. 2, pp. 339-353, 2012.

[19] D. Bianchi, A. Buccini, M. Donatelli, and S. Serra-Capizzano, "Iterated fractional Tikhonov regularization," PAMM, vol. 15, no. 1, pp. 581-582, 2015.

[20] D. Bianchi and M. Donatelli, "On generalized iterated Tikhonov regularization with operator-dependent seminorms," Electronic Transactions on Numerical Analysis, vol. 47, pp. 73-99, 2017.

[21] M. E. Hochstenbach and L. Reichel, "Fractional Tikhonov regularization for linear discrete ill-posed problems," BIT Numerical Mathematics, vol. 51, no. 1, pp. 197-215, 2011.

[22] R. H. Chan, A. Lanza, S. Morigi, and F. Sgallari, "An adaptive strategy for the restoration of textured images using fractional order regularization," Numerical Mathematics: Theory, Methods and Applications, vol. 6, no. 1, pp. 276-296, 2013.

[23] M. D. Ruiz-Medina, J. M. Angulo, and V. V. Anh, "Fractionalorder regularization and wavelet approximation to the inverse estimation problem for random fields," Journal of Multivariate Analysis, vol. 85, no. 1, pp. 192-216, 2003.

[24] J. Zhang, Z. Wei, and L. Xiao, "A fast adaptive reweighted residual-feedback iterative algorithm for fractional-order total variation regularized multiplicative noise removal of partlytextured images," Signal Processing, vol. 98, pp. 381-395, 2014.

[25] A. Y. Al-Bayati and R. S. Muhammad, "New scaled sufficient descent conjugate gradient algorithm for solving unconstraint optimization problems," Journal of Computer Science, vol. 6, no. 5, pp. 511-518, 2010.

[26] W. W. Hager and H. Zhang, "The limited memory conjugate gradient method," SIAM Journal on Optimization, vol. 23, no. 4, pp. 2150-2168, 2013.

[27] Y. J. Wang, C. Y. Wang, and N. H. Xiu, "A family of supermemory gradient projection methods for constrained optimization," Optimization. A Journal of Mathematical Programming and Operations Research, vol. 51, no. 6, pp. 889-905, 2002.

[28] K. W. Ng and A. Rohanin, "Solving optimal control problem of monodomain model using hybrid conjugate gradient methods," Mathematical Problems in Engineering, vol. 2012, Article ID 734070, 14 pages, 2012.

[29] Y. Narushima and H. Yabe, "Global convergence of a memory gradient method for unconstrained optimization," Computational optimization and applications, vol. 35, no. 3, pp. 325-346, 2006.

[30] J.-B. Jian, Y.-F. Zeng, and C.-M. Tang, "A generalized supermemory gradient projection method of strongly sub-feasible directions with strong convergence for nonlinear inequality constrained optimization," Computers \& Mathematics with Applications, vol. 54, no. 4, pp. 507-524, 2007.

[31] Y. Jia, Z. Yang, N. Guo, and L. Wang, "Random dynamic load identification based on error analysis and weighted total least squares method," Journal of Sound and Vibration, vol. 358, pp. 111-123, 2015.

[32] J. Liu, X. Sun, X. Han, C. Jiang, and D. Yu, "Dynamic load identification for stochastic structures based on Gegenbauer polynomial approximation and regularization method," Mechanical Systems and Signal Processing, vol. 56, pp. 35-54, 2015.

[33] T. Aktosun and C. van der Mee, "Solution of the inverse scattering problem for the three-dimensional Schroedinger equation using a Fredholm integral equation," SIAM Journal on Mathematical Analysis, vol. 22, no. 3, pp. 717-731, 2006.

[34] J. N. Franklin, "Minimum principles for ill-posed problems," SIAM Journal on Mathematical Analysis, vol. 9, no. 4, pp. 638650, 1978.

[35] N. N. Abdelmalek, "An algorithm for the solution of ill-posed linear systems arising from the discretization of Fredholm integral equation of the first kind," Journal of Mathematical Analysis and Applications, vol. 97, no. 1, pp. 95-111, 1983.

[36] G. L. Mazzieri and R. D. Spies, "Regularization methods for illposed problems in multiple Hilbert scales," Inverse Problems, vol. 28, no. 5, 055005, 30 pages, 2012.

[37] V. A. Morozov, "Application of the regularization method to the solution of an ill-posed problem," Proceedings of the Royal Society of Medicine, vol. 18, no. 4, pp. 401-406, 1965.

[38] N. H. Tuan and D. D. Trong, "A simple regularization method for the ill-posed evolution equation," Czechoslovak Mathematical Journal, vol. 61(136), no. 1, pp. 85-95, 2011.

[39] F. Stout and J. H. Kalivas, "Tikhonov regularization in standardized and general form for multivariate calibration with application towards removing unwanted spectral artifacts," Journal of Chemometrics, vol. 20, no. 1-2, pp. 22-33, 2006.

[40] Y. Wei, P. Xie, and L. Zhang, "Tikhonov Regularization and Randomized GSVD," Siam Journal on Matrix Analysis and Applications, vol. 37, no. 2, pp. 649-675, 2016.

[41] S. C. Omowunmi and A. A. Susu, "Application of Tikhonov Regularization Technique to the Kinetic Data of an Autocatalytic Reaction: Pyrolysis of N-Eicosane," Engineering Journal, vol. 03, no. 12, pp. 1161-1170, 2011.

[42] M. Z. Nashed, "The theory of Tikhonov regularization for fredholm equations of the first kind (C. W. Groetsch)," Siam Review, vol. 28, no. 1, pp. 116-118, 2006.

[43] H. Liu, X. Wang, and C. Lu, "Rolling bearing fault diagnosis under variable conditions using Hilbert-Huang transform and singular value decomposition," Mathematical Problems in Engineering, vol. 2014, Article ID 765621, 10 pages, 2014.

[44] G. M. Vainikko, "The discrepancy principle for a class of regularization methods," USSR Computational Mathematics and Mathematical Physics, vol. 22, no. 3, pp. 1-19, 1982.

[45] V. Albani, A. De Cezaro, and J. P. Zubelli, "On the choice of the Tikhonov regularization parameter and the discretization level: a discrepancy-based strategy," Inverse Problems and Imaging, vol. 10, no. 1, pp. 1-25, 2016.

[46] C. S. Liu, "Novel algorithms based on the conjugate gradient method for inverting ill-conditioned matrices, and a new regularization method to solve ill-posed linear systems," Computer Modeling in Engineering and Sciences, vol. 60, no. 3, pp. 279-308, 2010.

[47] C.-S. Liu, "An optimally generalized steepest-descent algorithm for solving ill-posed linear systems," Journal of Applied Mathematics, vol. 2013, Article ID 154358, 15 pages, 2013.

[48] C.-S. Liu, "Modifications of steepest descent method and conjugate gradient method against noise for ill-posed linear systems," Communications in Numerical Analysis, vol. 2012, Article ID cna-00115, 24 pages, 2012.

[49] G. Zoutendijk, "Nonlinear programming, computational methods," in Integer and Nonlinear Programming, pp. 37-86, NorthHolland, Amsterdam, The Netherlands, 1970.

[50] P. Wolfe, "Convergence conditions for ascent methods," SIAM Review, vol. 11, pp. 226-235, 1969. 
[51] P. Wolfe, "Convergence Conditions for Ascent Methods II: Some Corrections," Siam Review, vol. 13, no. 2, pp. 226-235, 2006.

[52] M. P. Baldwin and T. J. Dunkerton, "Stratospheric harbingers of anomalous weather regimes," Science, vol. 294, no. 5542, pp. 581-584, 2001.

[53] C.-S. Liu, C.-P. Ren, and D.-G. Li, "Reconstruction and deduction of cutting coal and rock load spectrum on modified discrete regularization algorithm," Journal of China Coal Society, vol. 39, no. 5, pp. 981-986, 2014.

[54] C. S. Liu, C. P. Ren, and F. Han, "Study on time-frequency spectrum characteristic of dynamic cutting load based on wavelet regularization," Applied Mechanics and Materials, vol. 577, pp. 196-200, 2014.

[55] C. Ren, N. Wang, and C. Liu, "Identification of random dynamic force using an improved maximum entropy regularization combined with a novel conjugate gradient," Mathematical Problems in Engineering, vol. 2017, Article ID 9125734, 14 pages, 2017. 


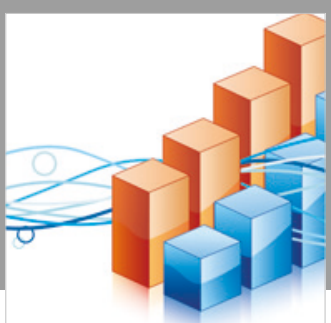

Advances in

Operations Research

\section{-n-m}
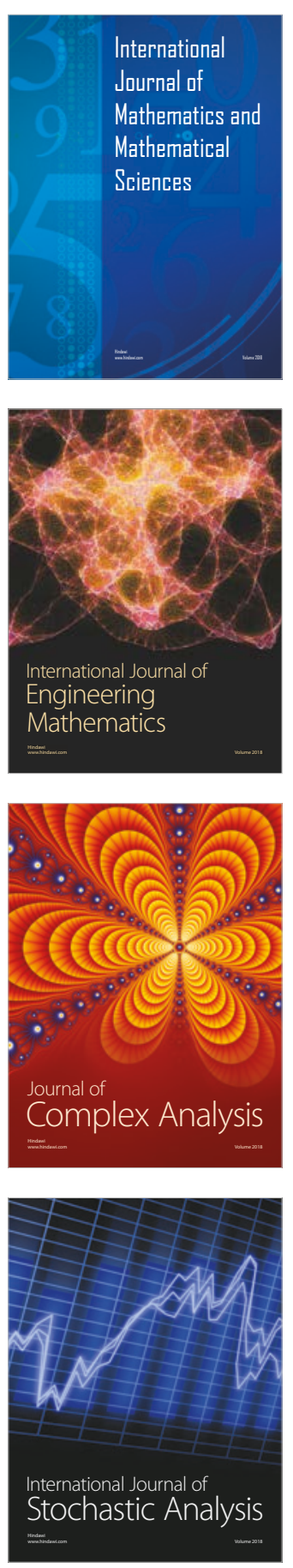
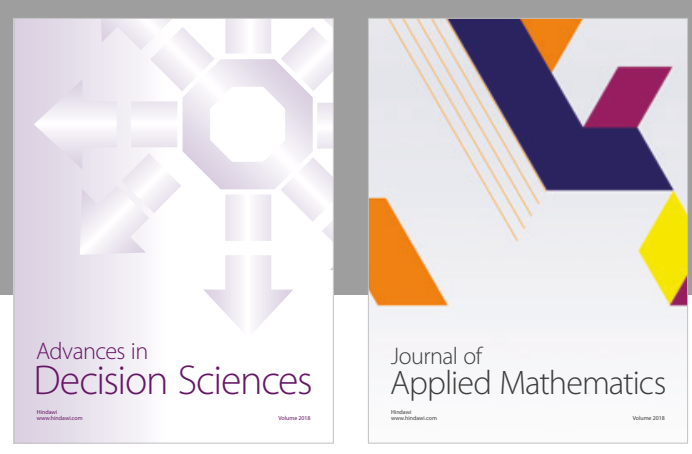

Journal of

Applied Mathematics
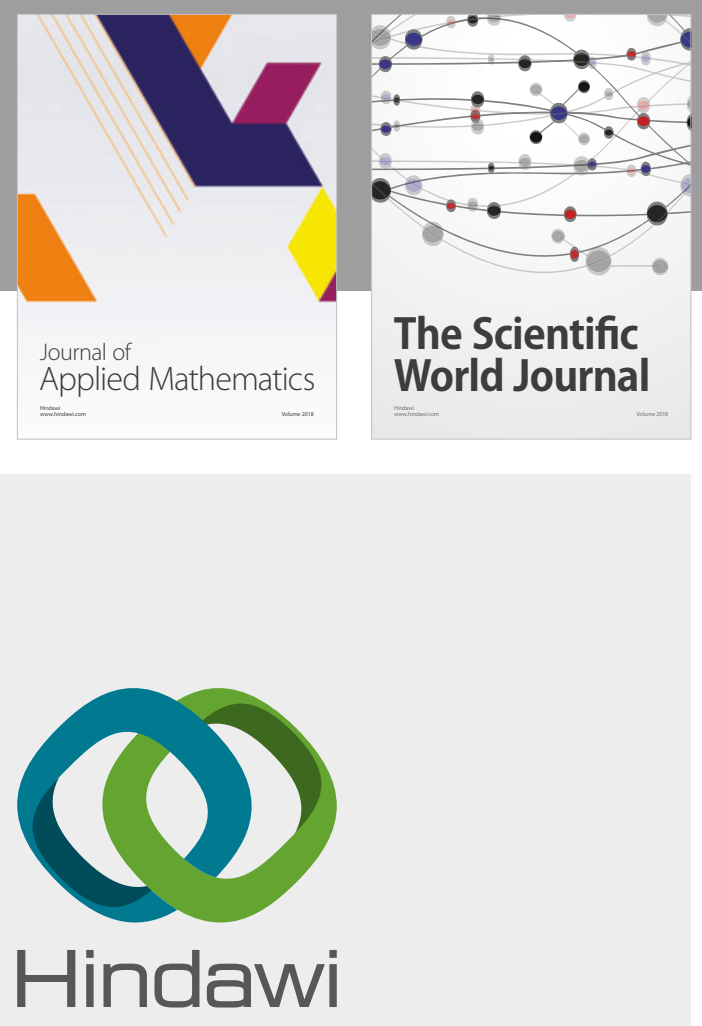

Submit your manuscripts at

www.hindawi.com

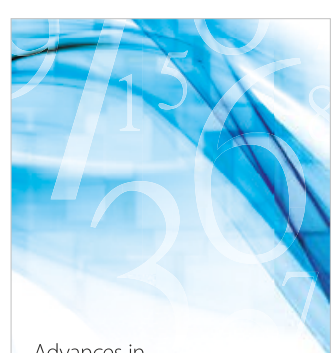

Advances in
Numerical Analysis
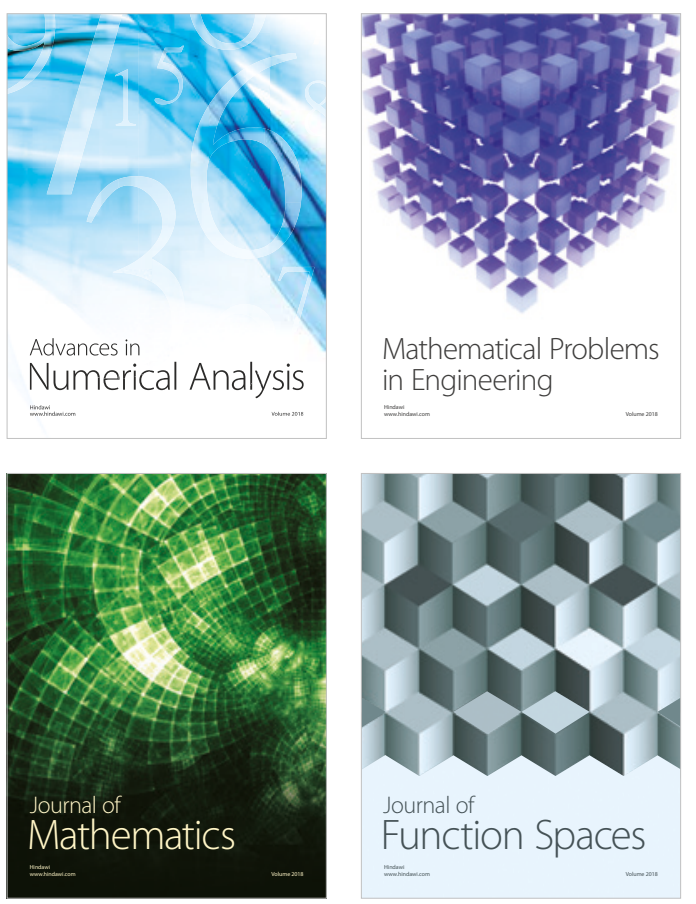

Mathematical Problems in Engineering

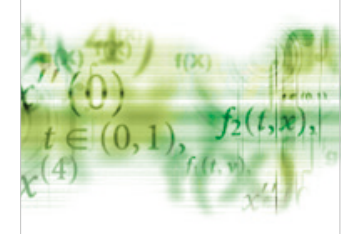

International Journal of

Differential Equations

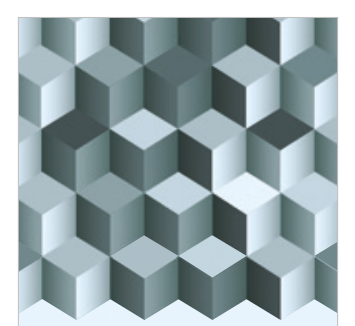

Journal of

Function Spaces

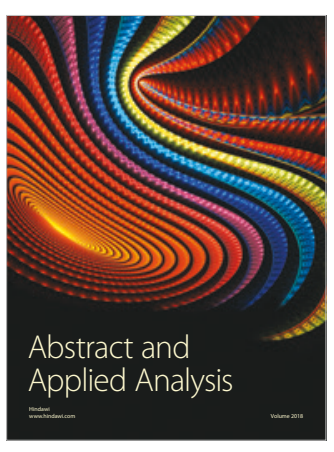

The Scientific

World Journal

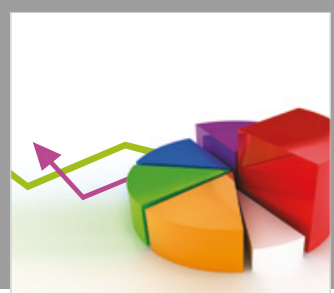

Journal of

Probability and Statistics
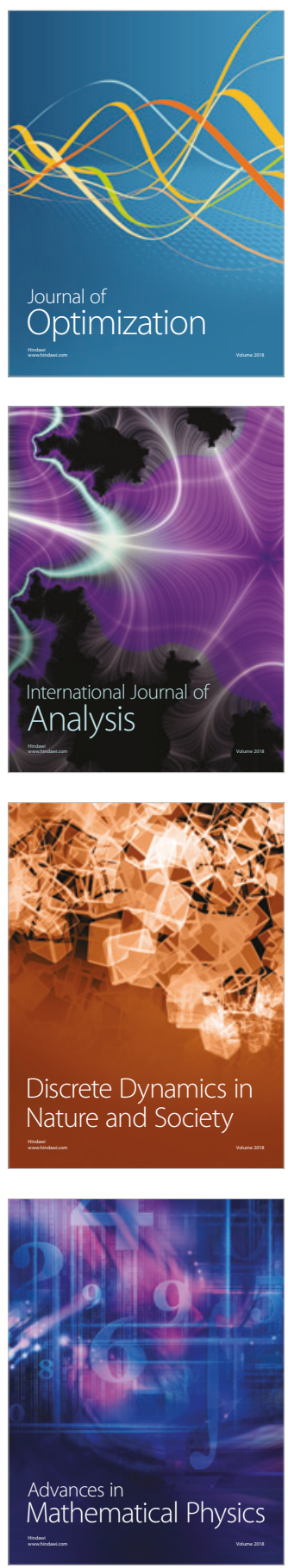